\title{
On convex relaxations of quadrilinear terms
}

\author{
Sonia Cafieri* $\quad$ Jon Lee ${ }^{\dagger} \quad$ Leo Liberti ${ }^{\ddagger}$
}

29th April 2009

\begin{abstract}
The best known method to find exact or at least $\varepsilon$-approximate solutions to polynomial programming problems is the spatial Branch-and-Bound algorithm, which rests on computing lower bounds to the value of the objective function to be minimized on each region that it explores. These lower bounds are often computed by solving convex relaxations of the original program. Although convex envelopes are explicitly known (via linear inequalities) for bilinear and trilinear terms on arbitrary boxes, such a description is unknown, in general, for multilinear terms of higher order. In this paper, we study convex relaxations of quadrilinear terms. We exploit associativity to rewrite such terms as products of bilinear and trilinear terms. Using a general technique, we establish that, any relaxation for $k$-linear terms that employs a successive use of relaxing bilinear terms (via the bilinear convex envelope) can be improved by employing instead a relaxation of a trilinear term (via the trilinear convex envelope). We present a computational analysis which helps establish which relaxations are strictly tighter, and we apply our findings to two well-studied applications: the Molecular Distance Geometry Problem and the Hartree-Fock Problem.

Keywords: quadrilinear, trilinear, bilinear, convex relaxation, reformulation, global optimization, spatial Branch and Bound, MINLP.
\end{abstract}

\section{Introduction}

A polynomial programming problem is a Nonlinear Program (NLP) in the following general form:

$$
\begin{array}{ll}
\min & f(x) \\
& g(x) \leq 0 \\
& x \in\left[x^{L}, x^{U}\right],
\end{array}
$$

where $f: \mathbb{R}^{n} \rightarrow \mathbb{R}, g: \mathbb{R}^{n} \rightarrow \mathbb{R}^{m}$ are polynomial functions, and $x^{L}, x^{U} \in \mathbb{R}^{n}$. In general, the feasible region of (1) can be a nonconvex set, or the objective function may be nonconvex on the feasible region; it is such instances that are primarily of interest.

The solution method of choice is the spatial Branch-and-Bound (sBB) algorithm [33, 2, 37, 21], which finds, in general, $\varepsilon$-approximate solutions for an arbitrarily small positive $\varepsilon$. The sBB algorithm works by recursively partitioning the search space (normally a box defined by variable ranges) along the coordinate direction that contributes most to the gap between lower and upper bounds on the optimal objective function value computed in each subproblem. For a minimization problem, the lower bound is usually computed by constructing and solving a convex relaxation, and the upper bound can simply be a local optimum found by a (local) NLP solver. When the gap for a particular subproblem is within $\varepsilon$, the

\footnotetext{
*LIX, École Polytechnique, 91128 Palaiseau, France. cafieri@lix.polytechnique.fr

$\dagger$ Dept. of Mathematical Sciences, IBM T.J. Watson Research Center, PO Box 218, Yorktown Heights, N.Y. 10598, USA. jonlee@us.ibm.com

${ }^{\ddagger}$ LIX, École Polytechnique, 91128 Palaiseau, France. liberti@lix.polytechnique.fr
} 
subproblem is discarded without further partitioning, because the globally optimal objective value for that subproblem has been found. Subproblems are also discarded when the convex relaxation is infeasible or when their lower bound is greater than the current best overall solution found. The algorithm terminates when all the subproblems have been discarded.

One of the most crucial steps of $\mathrm{sBB}$ is the lower bound computation. For factorable problems (i.e. NLPs involving functional forms that can be written as recursively, using a finite number of elementary "atomic" functions), it is possible to construct a convex relaxation automatically by means of a particular type of lifting reformulation (called MINLP standard form [37, 22]) first proposed in [28] and then exploited in most existing sBB algorithms [33, 2, 37, 21, 40, 5]. Because all polynomial functions are factorable (relative to multiplication), such a reformulation also applies to (1). Informally, high-order monomials are recursively rewritten as products of monomials of sufficiently low order for which a tight convex relaxation (possibly the convex envelope) is known. Each low-order monomial is replaced by an additional variable, and an equality constraint defining the additional variable in terms of the monomial it replaces is adjoined to (1). This operation is carried out recursively, until the functions $f, g$ are linear forms. At this stage, each defining constraint is replaced by a set of constraints defining the convex relaxation of its feasible set, thus yielding a convex relaxation for the whole problem.

This iterative procedure for constructing a convex relaxation of (1) is symbolic rather than numeric, in the sense that it performs structural changes to the formulation of (1), adjoining variables and constraints, and replacing terms with variables and constraints with other constraints. The tightness of the resulting relaxation rests on the availability of a good "library" of convex relaxations/envelopes of elementary terms, such as monomials of low degree. Notice that this symbolic procedure is independent of any geometrical consideration concerning the feasible set of (1); thus, the direction of the objective function at a point is not an option for measuring convex relaxation tightness; we employ the partial order of set containment instead: convex relaxation $A$ is tighter than convex relaxation $B$ if $A \subsetneq B$.

Convex envelopes in explicit form are currently known for concave/convex univariate functions [1, 36], bilinear terms [3, 28], trilinear terms [30, 29], univariate monomials of odd degree [20, 26] and fractional terms [39]. General theoretical results for vertex-polyhedral convex envelopes are given in [38]. More advanced practical techniques for generating tight convex envelopes computationally are given in [11, 12]. The multivariate monomial of smallest degree for which the convex envelope is not known in general is the quartic one [7]. In [34], bounding schemes for multilinear functions are compared; convex envelopes of multilinear terms are known for specific values of $x^{L}, x^{U}[32]$; a recent result characterizes the convex envelope of a class of functions including some multilinear functions [14]. The general quartic terms, up to symmetry on variable indices, are $x_{1} x_{2} x_{3} x_{4}, x_{1} x_{2} x_{3}^{2}, x_{1} x_{2}^{3}, x_{1}^{2} x_{2}^{2}$. Besides being fundamental building blocks for reformulating the general problem (1), these terms occur in important applications, such as the Molecular Distance Geometry Problem (MDGP) (see [16, 17, 23]) and the Hartree-Fock Problem (HFP) $($ see $[18,24])$.

In this paper, we focus on the quadrilinear term $x_{1} x_{2} x_{3} x_{4}$ (other types of quartic terms will be the object of future investigations). Associativity allows the iterative procedure to decomopose this term in several different ways: $\left(\left(x_{1} x_{2}\right) x_{3}\right) x_{4}$ and $\left(x_{1} x_{2} x_{3}\right) x_{4}$ are two examples yielding as different outcomes the set of defining constraints $w_{1}=x_{1} x_{2}, w_{2}=w_{1} x_{3}, w_{3}=w_{2} x_{4}$ and, respectively, $w_{1}=x_{1} x_{2} x_{3}, w_{2}=w_{1} x_{4}$ (where the $w$ are added variables). It is this flexibility that we wish to investigate. We prove, using a general method that is not limited to monomials, that the second alternative yields a relaxation that is at least as tight as the first. Also, we establish by means of a computational assessment, that in many cases the second alternative yields strictly tighter relaxations. Finally, we study the behaviour of the proposed relaxations when used for the MDGP and HFP.

The results that we obtain are important especially in view of the fact that the traditional grouping used by sBB algorithms $[2,35,27,5]$ is the slacker alternative $\left(\left(x_{1} x_{2}\right) x_{3}\right) x_{4}$. Moreover, because the symbolic-relaxation procedure illustrated above also holds in the presence of integrality constraints on the variable vector $x$, and even when $f, g$ include transcendental terms such as logarithm, exponentials and trigonometric functions, our results also apply to rather general Mixed-Integer Nonlinear Programming (MINLP) problems. 
The rest of this paper is organized as follows. In $\S 2$ we review the explicit convex (linear) envelopes for bilinear and trilinear terms, and we use them to derive explicit convex (linear) relaxations of a quadrilinear term. In $\S 3$ we define a small formal language for expressing functions in infix form, and describe language semantics corresponding to the function itself and its reformulation/relaxation. We then exploit these formalisms to prove our main result. In $\S 4$ we describe an experimental methodology to determine which type of term grouping order is best for the quadrilinear term. In $\S 5$ we introduce the MDGP and the HFP, and we report the results of computational experiments aimed at comparing different convex relaxations on application models. $\S 6$ concludes the paper.

\section{Convex relaxations of quadrilinear terms}

\subsection{Existing convex envelopes}

In this section, we review the known convex envelopes for bilinear and trilinear terms that we use to derive convex relaxations for the quadrilinear term. Each of these should be considered as a symbolic algorithm to be applied to the original problem in order to obtain a (convex) relaxation. Throughout, the domain of each variable $x_{i}$ is the interval denoted $\left[x_{i}^{L}, x_{i}^{U}\right]$.

The bilinear term $x_{j} x_{k}$ is replaced by a new variable $x_{i}$, and the following linear inequalities are added to the problem relaxation ("McCormick's envelope"; see [3, 28]):

$$
\begin{aligned}
& x_{i} \geq x_{j}^{L} x_{k}+x_{k}^{L} x_{j}-x_{j}^{L} x_{k}^{L} \\
& x_{i} \geq x_{j}^{U} x_{k}+x_{k}^{U} x_{j}-x_{j}^{U} x_{k}^{U} \\
& x_{i} \leq x_{j}^{L} x_{k}+x_{k}^{U} x_{j}-x_{j}^{L} x_{k}^{U} \\
& x_{i} \leq x_{j}^{U} x_{k}+x_{k}^{L} x_{j}-x_{j}^{U} x_{k}^{L} .
\end{aligned}
$$

The trilinear term $x_{j} x_{k} x_{h}$ is replaced by a new variable $x_{i}$, and linear inequalities describing the convex envelope are added to the problem relaxation depending on the signs of the bounds on variables [30, 29]. Denoting a permutation of $x_{j}, x_{k}, x_{h}$ by the symbols $x, y, z$, in the case $x^{L} \geq 0, y^{L} \geq 0, z^{L} \leq 0, z^{U} \geq 0$ the following inequalities are added:

$$
\begin{aligned}
x_{i} \geq y^{U} z^{U} x+x^{U} z^{U} y+x^{U} y^{U} z-2 x^{U} y^{U} z^{U} \\
x_{i} \geq y^{U} z^{L} x+x^{L} z^{U} y+x^{L} y^{U} z-x^{L} y^{U} z^{L}-x^{L} y^{U} z^{U} \\
x_{i} \geq y^{U} z^{L} x+x^{L} z^{L} y+x^{L} y^{L} z-x^{L} y^{U} z^{L}-x^{L} y^{L} z^{L} \\
x_{i} \geq y^{L} z^{U} x+x^{U} z^{L} y+x^{U} y^{L} z-x^{U} y^{L} z^{U}-x^{U} y^{L} z^{L} \\
x_{i} \geq y^{L} z^{L} x+x^{U} z^{L} y+x^{L} y^{L} z-x^{U} y^{L} z^{L}-x^{L} y^{L} z^{L} \\
x_{i} \geq y^{L} z^{U} x+x^{L} z^{U} y+\left(\theta /\left(z^{U}-z^{L}\right)\right) z+ \\
\\
x_{i} \leq\left(-\left(\theta z^{L}\right) /\left(z^{U}-z^{L}\right)-x^{L} y^{U} z^{U}-x^{U} y^{L} z^{U}+x^{U} y^{U} z^{L}\right) \\
x_{i} \leq y^{L} z^{L} x+x^{U} z^{U} y-x^{U} y^{U} z-2 x^{U} y^{U} z^{L} \\
x_{i} \leq y^{U} y^{L} z^{U}-x^{U} y^{L} z^{L} \\
x_{i} \leq x^{L} z^{U} y+x^{L} y^{L} z-x^{L} y^{U} z^{U}-x^{L} y^{L} z^{U} \\
x_{i} \leq x^{L} z^{L} y+x^{L} y^{U} z-x^{L} y^{U} z^{U}-x^{L} y^{U} z^{L} \\
x_{i} \leq x^{L} z^{U} y+x^{L} y^{L} z-x^{U} y^{L} z^{U}-x^{L} y^{L} z^{U} \\
\end{aligned}
$$

where

$$
\begin{aligned}
\theta & :=x^{L} y^{U} z^{U}-x^{U} y^{U} z^{L}-x^{L} y^{L} z^{U}+x^{U} y^{L} z^{U} \\
\bar{\theta} & :=x^{U} y^{L} z^{L}-x^{U} y^{U} z^{U}-x^{L} y^{L} z^{L}+x^{L} y^{U} z^{L}
\end{aligned} .
$$


For details about other cases, depending on the signs of the bounds, the reader is referred to [30, 29], where explicit expressions defining the facets of convex/concave envelopes are given for different combinations of variable bounds.

\subsection{Obtaining convex relaxations of quadrilinear terms}

Let us consider a quadrilinear term $x_{1} x_{2} x_{3} x_{4}$. Exploiting associativity of the product, we rewrite it, in different ways, as products of monomials of degree two and three and use the known bilinear and trilinear convex envelopes (described in the previous subsection) to derive the corresponding convex relaxations.

We consider the four types of term groupings

$$
\begin{array}{r}
\left(\left(x_{1} x_{2}\right) x_{3}\right) x_{4} \\
\left(x_{1} x_{2}\right)\left(x_{3} x_{4}\right) \\
\left(x_{1} x_{2} x_{3}\right) x_{4} \\
\left(x_{1} x_{2}\right) x_{3} x_{4},
\end{array}
$$

which, up to renaming the variables, exhausts the possibilities. Relative to these groupings, we consider, respectively, the sets:

$$
\begin{aligned}
& S_{1}=\left\{(x, w) \in \mathbb{R}^{4} \times \mathbb{R}^{3} \mid x_{i} \in\left[x_{i}^{L}, x_{i}^{U}\right], w_{1}=x_{1} x_{2}, w_{2}=w_{1} x_{3}, w_{3}=w_{2} x_{4}\right\}, \\
& S_{2}=\left\{(x, w) \in \mathbb{R}^{4} \times \mathbb{R}^{3} \mid x_{i} \in\left[x_{i}^{L}, x_{i}^{U}\right], w_{1}=x_{1} x_{2}, w_{2}=x_{3} x_{4}, w_{3}=w_{1} w_{2}\right\}, \\
& S_{3}=\left\{(x, w) \in \mathbb{R}^{4} \times \mathbb{R}^{2} \mid x_{i} \in\left[x_{i}^{L}, x_{i}^{U}\right], w_{1}=x_{1} x_{2} x_{3}, w_{2}=w_{1} x_{4}\right\}, \\
& S_{4}=\left\{(x, w) \in \mathbb{R}^{4} \times \mathbb{R}^{2} \mid x_{i} \in\left[x_{i}^{L}, x_{i}^{U}\right], w_{1}=x_{1} x_{2}, w_{2}=w_{1} x_{3} x_{4}\right\} .
\end{aligned}
$$

To derive four corresponding relaxations, we exploit a bilinear envelope thrice for the first two cases; a trilinear envelope followed by a bilinear envelope for $S_{3}$ and a bilinear envelope followed by a trilinear envelope for $S_{4}$.

\section{Main result}

In this section, we provide a theoretical framework to investigate relaxation strength. We point out that it can be applied to any factorable mathematical program in order to compare pairs of relaxations. The general method that we propose is based on the idea of using a formal language to express the functions used in the objective and constraints of a mathematical program, and defining a semantic of strings of this language that is used to prove the main result.

A list of symbols used in this section, together with their meaning, is reported in Table 1.

\subsection{Mathematical expression language}

Let $\mathscr{X}=\left\{x_{1}, \ldots, x_{n}\right\}$ be a set of variable symbols. Let $\mathscr{O}$ be a set of operator symbols. Operators are written in functional form, i.e. for an operator $\otimes \in \mathscr{O}$ with $p \geq 1$ arguments, we let $\otimes\left(x_{1}, \ldots, x_{p}\right)$ be the string describing the application of the operator $\otimes$ to the formal arguments $x_{1}, \ldots, x_{p}$ (sometimes, depending on the context, we also write the more usual infix form $x_{1} \otimes \cdots \otimes x_{p}$ ). For an operator $\otimes \in \mathscr{O}$, we let the arity $\alpha(\otimes)$ be the set of numbers of arguments that the operator $\otimes$ can have. Let $\mathscr{P}$ be the set comprising the three symbols: "(", ")" and "," — that is: left round bracket, right round bracket, and comma.

Consider the alphabet $\mathscr{A}=\mathscr{X} \cup \mathbb{R} \cup \mathscr{O} \cup \mathscr{P}$, which we use to define a language whose strings (i.e. valid words) are precisely the functions used in the objective and constraints of a mathematical program. 


\begin{tabular}{||c|l||}
\hline \hline Symbol & Meaning \\
\hline $\mathscr{X}$ & set of variables symbols \\
$\mathscr{O}$ & set of operator symbols \\
$\mathscr{P}$ & set of round brackets and comma symbols \\
$\mathscr{A}$ & alphabet \\
$\mathscr{L}$ & language \\
$f, h$ & strings of the language \\
$\beta_{f}(i)$ & symbol of the string $f$ in the $i$-th position \\
$\omega(\otimes)$ & result of application of the operator $\otimes$ (to elements of the language) \\
$\mathscr{S}(f)$ & semantic of $f$ \\
$\mathscr{R}(f)$ & relaxed semantic of $f$ \\
$R(h)$ & relaxed composite semantic of $h$ with respect to its substring \\
\hline \hline
\end{tabular}

Table 1: Table of notations

\subsection{Example}

For $\mathscr{O}=\{+,-, \times, \div, \uparrow, \sqrt{ }, \log , \exp , \sin , \cos , \tan \}$, we have the arities:

$$
\begin{aligned}
\alpha(+) & =\mathbb{N} \\
\alpha(-) & =\{1,2\} \\
\alpha(\times) & =\mathbb{N} \\
\alpha(\div) & =\alpha(\uparrow)=\{2\} \\
\alpha(\sqrt{)} & =\alpha(\log )=\alpha(\exp )=\alpha(\sin )=\alpha(\cos )=\alpha(\tan )=\{1\} .
\end{aligned}
$$

The function $-\sin \left(x^{2}-\frac{\sqrt{x}(y+1)(y-2)}{\log y}\right)$ is given by the following string:

$$
-(\sin (-(\uparrow(x, 2)), \div(\times(\sqrt{(} x),+(y, 1),-(y, 2)), \log (y)))) .
$$

Let $\mathscr{L}$ be the language (set of strings of $\mathscr{A}$ ) built recursively according to the following rules: atomic expressions consisting of a single variable or real number are in the language, and for every operator and potential arity, if the arity $p$ is compatible with the operator, then by applying the operator to $p$ (ordered) elements of the language, we get another element of the language:

1. $\forall \ell \in \mathbb{R} \cup \mathscr{X}(\ell \in \mathscr{L})$

2. $\forall \otimes \in \mathscr{O}, p \in \mathbb{N}\left(p \in \alpha(\otimes) \rightarrow \forall \ell_{1} \ldots, \ell_{p} \in \mathscr{L}\left(\otimes\left(\ell_{1}, \ldots, \ell_{p}\right) \in \mathscr{L}\right)\right)$.

In the latter case, we also write $\omega(\otimes)=\left(\ell_{1}, \ldots, \ell_{p}\right)$. For $f \in \mathscr{L}$, we write $f\left(x_{i_{1}}, \ldots, x_{i_{p}}\right)$ to emphasize the fact that the string for $f$ only includes variable symbols $x_{i_{j}}$ for $j \leq p$.

\subsection{Relaxed semantics}

We now introduce the formal definition of relaxed semantic of strings in $\mathscr{L}$.

Let $x \in \mathbb{R}^{n}$ be such that $x^{L} \leq x \leq x^{U}$ for $x^{L}, x^{U} \in \mathbb{R}^{n}$, and let $f \in \mathscr{L}$. Consider the sets:

$$
\begin{aligned}
& \mathscr{S}(f)=\left\{\left(w_{f}, x\right) \mid w_{f}=f(x), x^{L} \leq x \leq x^{U}\right\} \\
& \mathscr{R}(f)=\left\{\left(w_{f}, x\right) \mid A_{f}\left(w_{f}, x\right) \leq b_{f}, x^{L} \leq x \leq x^{U}\right\},
\end{aligned}
$$

where $b_{f} \in \mathbb{R}^{m}, A_{f}\left(w_{f}, x\right): \mathbb{R}^{(n+1)} \rightarrow \mathbb{R}^{m}$ is a convex function, and $\mathscr{S}(f) \subseteq \mathscr{R}(f)$. We call $\mathscr{R}(f)$ a relaxed semantic of $f$.

We also consider a relaxed semantic over substrings of $f$. For all $i \leq p$, let $f_{i}, g, h \in \mathscr{L}$ be such that $h(x)=g\left(f_{1}(x), \ldots, f_{p}(x)\right)$. Let $\mathbf{w}_{f}=\left(w_{f_{1}}, \ldots, w_{f_{p}}\right), \mathbf{w}=\left(w_{1}, \ldots, w_{p}\right)$, and consider sets

$$
\begin{aligned}
& \bar{R}(h)=\left\{\left(w_{g}, \mathbf{w}_{f}, x\right) \mid A_{g}\left(w_{g}, \mathbf{w}_{f}\right) \leq b_{g}, A_{f_{i}}\left(w_{f_{i}}, x\right) \leq b_{f_{i}} \forall i \leq p, x^{L} \leq x \leq x^{U}\right\} \\
& R(h)=\{(w, x) \mid \exists \mathbf{w}(w, \mathbf{w}, x) \in \bar{R}(h)\},
\end{aligned}
$$


where $R(h)$ is the projection of $\bar{R}(h)$ on the subspace $\left\{(w, x) \in \mathbb{R}^{n+1}\right\} . R(h)$ is the relaxed composite semantic of $h$ with respect to its substring $g\left(f_{1}, \ldots, f_{p}\right)$.

In what follows, we always assume that $\mathscr{R}(h) \subseteq R(h)$, i.e. the relaxed semantic is at least as tight as the relaxed composite semantic.

Note that it is possible to define relaxed semantics slacker than relaxed composite semantics.

\subsection{Example}

Let $x=\left(x_{1}, x_{2}, x_{3}\right) \in \mathbb{R}^{3}, x^{L}=\left(x_{1}^{L}, x_{2}^{L}, x_{3}^{L}\right)$ and $x^{U}=\left(x_{1}^{U}, x_{2}^{U}, x_{3}^{U}\right)$ be such that $x^{L} \leq x \leq x^{U}$, where $x^{L}=-10$ and $x^{U}=5$.

Consider the relaxed semantic given by the application of interval arithmetic. We recall that, for two intervals $[a, b]$ and $[b, c]$ such that $a \leq 0 \leq b, c \leq 0 \leq d$, the application of basic interval arithmetic rules gives the interval $[\min (b c, a d), \max (a c, b d)]$. Hence, applying interval arithmetic on $x_{1} x_{2} x_{3}$, we get the hyperrectangle $R_{i a}$ given by $[-10,5] \times[-10,5] \times[-10,5] \times[-1000,500]$.

As relaxed composite semantic, we consider that obtained by applying the bilinear convex relaxation twice. Let $w_{1}=x_{1} x_{2}$ and $w_{2}=w_{1} x_{3}$. We have:

$$
\begin{aligned}
\bar{R}_{b}= & \left\{\left(w_{1}, w_{2}, x\right) \in \mathbb{R}^{5} \mid x^{L} \leq x \leq x^{U},\right. \\
& w_{1} \geq x_{1}^{L} x_{2}+x_{2}^{L} x_{1}-x_{1}^{L} x_{2}^{L}, w_{1} \geq x_{1}^{U} x_{2}+x_{2}^{U} x_{1}-x_{1}^{U} x_{2}^{U}, \\
& w_{1} \leq x_{1}^{L} x_{2}+x_{2}^{U} x_{1}-x_{1}^{L} x_{2}^{U}, w_{1} \leq x_{1}^{U} x_{2}+x_{2}^{L} x_{1}-x_{1}^{U} x_{2}^{L}, \\
& w_{2} \geq w_{1}^{L} x_{3}+x_{3}^{L} w_{1}-w_{1}^{L} x_{3}^{L}, w_{2} \geq w_{1}^{U} x_{3}+x_{3}^{U} w_{1}-w_{1}^{U} x_{3}^{U}, \\
& \left.w_{2} \leq w_{1}^{L} x_{3}+x_{3}^{U} w_{1}-w_{1}^{L} x_{3}^{U}, w_{2} \leq w_{1}^{U} x_{3}+x_{3}^{L} w_{1}-w_{1}^{U} x_{3}^{L}\right\} .
\end{aligned}
$$

The polytope obtained projecting $\bar{R}_{b}$ on $\mathbb{R}^{4}$ has extreme points $(-10,-10,-10,-1000),(-10,-10,5,500)$, $(-10,5,-10,500),(5,-10,-10,500),(-10,5,5,-250),(5,-10,5,-250),(5,5,-10,-250),\left(5,5,-\frac{5}{2},-625\right)$, $\left(5,5,-\frac{5}{2}, 500\right),(5,5,5,125)$, that are contained in $R_{i a}$.

\subsection{Comparison of relaxed semantics}

Let $F \in \mathscr{L}$. Let $\mathscr{O}^{\prime}=\mathscr{O} \cup\{h\}$, where $h(x)=g\left(f_{1}(x), \ldots, f_{p}(x)\right)$, and let $\mathscr{A}^{\prime}=\mathscr{A}$. Let $F^{\prime}$ be $F$ rewritten using the rule $g\left(f_{1}, \ldots, f_{p}\right) \rightarrow h$, i.e. using the alphabet in $\mathscr{A}^{\prime}$.

\subsection{Theorem}

$R\left(F^{\prime}\right) \subseteq R(F)$.

Proof. Let $h$ be a string of the alphabet $\mathscr{A}$ such that $h(x)=g\left(f_{1}(x), \ldots, f_{p}(x)\right)$, and let $h^{\prime}$ be a string of the alphabet $\mathscr{A}^{\prime}$ written using the operator replacing $g\left(f_{1}, \ldots, f_{p}\right) \in \mathscr{L}$. The relaxed composite semantic of $F$ and of $F^{\prime}$ are given by:

$$
\begin{aligned}
& \bar{R}(F)=\left\{\left(w_{g}, \mathbf{w}_{f}, x\right) \mid A_{g}\left(w_{g}, \mathbf{w}_{f}\right) \leq b_{g}, \forall i \leq p A_{f_{i}}\left(w_{f_{i}}, x\right) \leq b_{f_{i}}, x^{L} \leq x \leq x^{U}\right\} \\
& \cup \bigcup_{k \leq t}\left\{\left(w_{s_{k}}, \mathbf{w}_{r_{k}}, x\right) \mid A_{s_{k}}\left(w_{s_{k}}, \mathbf{w}_{r_{k}}\right) \leq b_{s_{k}},\right. \\
&\left.\forall j \leq p A_{r_{j_{k}}}\left(w_{r_{j_{k}}}, x\right) \leq b_{r_{j_{k}}}, x^{L} \leq x \leq x^{U}\right\}, \\
& \bar{R}\left(F^{\prime}\right)=\quad\left\{\left(w_{h^{\prime}}, x\right) \mid A^{\prime}\left(w_{h^{\prime}}, x\right) \leq b^{\prime}, x^{L} \leq x \leq x^{U}\right\} \\
& \cup \bigcup_{k \leq t}\left\{\left(w_{s_{k}}, \mathbf{w}_{r_{k}}, x\right) \mid A_{s_{k}}\left(w_{s_{k}}, \mathbf{w}_{r_{k}}\right) \leq b_{s_{k}},\right. \\
&\left.\forall j \leq p A_{r_{j_{k}}}\left(w_{r_{j_{k}}}, x\right) \leq b_{r_{j_{k}}}, x^{L} \leq x \leq x^{U}\right\},
\end{aligned}
$$


where $\mathbf{w}_{f}=\left(w_{f_{1}}, \ldots, w_{f_{p}}\right), \forall k \leq t \mathbf{w}_{r_{k}}=\left(w_{r_{1}}, \ldots, w_{r_{p}}\right), w_{h^{\prime}}=h^{\prime}(x), p \geq 1$ and $t \geq 0$. Consider the relaxed composite semantic of $h$ and of $h^{\prime}$

$$
\begin{aligned}
\bar{R}(h) & =\left\{\left(w_{g}, \mathbf{w}_{f}, x\right) \mid A_{g}\left(w_{g}, \mathbf{w}_{f}\right) \leq b_{g}, \forall i \leq p A_{f_{i}}\left(w_{f_{i}}, x\right) \leq b_{f_{i}}, x^{L} \leq x \leq x^{U}\right\}, \\
\bar{R}\left(h^{\prime}\right) & =\left\{\left(w_{h^{\prime}}, x\right) \mid A^{\prime}\left(w_{h^{\prime}}, x\right) \leq b^{\prime}, x^{L} \leq x \leq x^{U}\right\},
\end{aligned}
$$

and their projections $R(h)=\{(w, x) \mid \exists \mathbf{w}(w, \mathbf{w}, x) \in \bar{R}(h)\}$ and similarly for $R\left(h^{\prime}\right)$. Furthermore, let $\mathscr{R}(h)$ be the relaxed semantic of $h$ :

$$
\mathscr{R}(h)=\left\{\left(w_{h}, x\right) \mid A_{h}\left(w_{h}, x\right) \leq b_{h}, x^{L} \leq x \leq x^{U}\right\}
$$

where $w_{h}=h(x)=g\left(f_{1}(x), \ldots, f_{p}(x)\right)$. By construction this is equal to $h^{\prime}(x)$, so that $\mathscr{R}(h) \equiv R\left(h^{\prime}\right)$. From the hypothesis that $\mathscr{R}(h) \subseteq R(h)$ it follows that $R\left(h^{\prime}\right) \subseteq R(h)$. The same inclusion holds in the lifted space: $\bar{R}\left(h^{\prime}\right) \subseteq \bar{R}(h)$. Because all the other terms $A_{s_{k}}\left(w_{s_{k}}, \mathbf{w}_{r_{k}}\right) \leq b_{s_{k}} \forall k \leq t$ are the same across both definitions, we have: $\bar{R}\left(F^{\prime}\right) \subseteq \bar{R}(F)$ and hence $R\left(F^{\prime}\right) \subseteq R(F)$.

Theorem 3.3 proves that for any relaxation of $x_{1} x_{2} \cdots x_{k}(k \geq 3)$ using any bilinear envelopes recursively, there is a relaxation employing also trilinear envelopes that is at least as tight. Moreover, such a relaxation can even be arrived at by replacing any single $(a \times b) \times c$ with $a \times b \times c$. In particular, if applied to the convex relaxations of $x_{1} x_{2} x_{3} x_{4}$ described in Section 2, the theorem proves that $S_{4}$ is at least as tight as $S_{1}$ and $S_{2}$, and that $S_{3}$ is at least as tight as $S_{1}$. Note that it does not give an indication on the relative tightness of $S_{3}$ and $S_{2}$. In the following, we show in detail how Theorem 3.3 can be applied to compare the tightness of two of the considered relaxations, namely $S_{1}$ and $S_{3}$.

\subsection{Example}

Let $x=\left(x_{1}, x_{2}, x_{3}, x_{4}\right) \in \mathbb{R}^{4}, x^{L}=\left(x_{1}^{L}, x_{2}^{L}, x_{3}^{L}, x_{4}^{L}\right)$ and $x^{U}=\left(x_{1}^{U}, x_{2}^{U}, x_{3}^{U}, x_{4}^{U}\right)$ be such that $x^{L} \leq x \leq x^{U}$. Let us consider a quadrilinear term $x_{1} x_{2} x_{3} x_{4}$ and the two term grouping $\left(\left(x_{1} x_{2}\right) x_{3}\right) x_{4}$ and $\left(x_{1} x_{2} x_{3}\right) x_{4}$. We assume for this example that $x^{L} \geq 0, y^{L} \geq 0, z^{L} \leq 0, z^{U} \geq 0$, where the symbols $x, y, z$ denote a permutation of $x_{1}, x_{2}, x_{3}$.

The sets of involved variable and operator symbols are $\mathscr{X}=\left\{x_{1}, x_{2}, x_{3}, x_{4}\right\}, \mathscr{O}=\{\times\}$ (we also write yz meaning $y \times z)$. Let $\mathscr{A}$ be the alphabet $\mathscr{A}=\mathscr{X} \cup \mathbb{R} \cup \mathscr{O} \cup \mathscr{P}$.

Consider first $\left(\left(x_{1} x_{2}\right) x_{3}\right) x_{4}$. Let $l, f, g, h \in \mathscr{L}$ be such that $h(x)=g(f(l(x)))$ and suppose that $l, f, g$ are such that $w_{l}=x_{1} x_{2}, w_{f}=w_{l} x_{3}$ and $w_{g}=w_{f} x_{4}$. We have:

$$
\begin{aligned}
\bar{R}(h)= & \left\{\left(w_{g}, w_{f}, w_{l}, x\right) \in \mathbb{R}^{7} \mid x^{L} \leq x \leq x^{U},\right. \\
& w_{l} \geq x_{1}^{L} x_{2}+x_{2}^{L} x_{1}-x_{1}^{L} x_{2}^{L}, w_{l} \geq x_{1}^{U} x_{2}+x_{2}^{U} x_{1}-x_{1}^{U} x_{2}^{U}, \\
& w_{l} \leq x_{1}^{L} x_{2}+x_{2}^{U} x_{1}-x_{1}^{L} x_{2}^{U}, w_{l} \leq x_{1}^{U} x_{2}+x_{2}^{L} x_{1}-x_{1}^{U} x_{2}^{L}, \\
& w_{f} \geq w_{l}^{L} x_{3}+x_{3}^{L} w_{l}-w_{l}^{L} x_{3}^{L}, w_{f} \geq w_{l}^{U} x_{3}+x_{3}^{U} w_{l}-w_{l}^{U} x_{3}^{U}, \\
& w_{f} \leq w_{l}^{L} x_{3}+x_{3}^{U} w_{l}-w_{l}^{L} x_{3}^{U}, w_{f} \leq w_{l}^{U} x_{3}+x_{3}^{L} w_{l}-w_{l}^{U} x_{3}^{L}, \\
& w_{g} \geq w_{f}^{L} x_{4}+x_{4}^{L} w_{f}-w_{f}^{L} x_{4}^{L}, w_{g} \geq w_{f}^{U} x_{4}+x_{4}^{U} w_{f}-w_{f}^{U} x_{4}^{U}, \\
& \left.w_{g} \leq w_{f}^{L} x_{4}+x_{4}^{U} w_{f}-w_{f}^{L} x_{4}^{U}, w_{g} \leq w_{f}^{U} x_{4}+x_{4}^{L} w_{f}-w_{f}^{U} x_{4}^{L}\right\} .
\end{aligned}
$$

The relaxed composite semantic $R(h)$ is given by the projection on the space of $(w, x) \in \mathbb{R}^{5}$.

Now consider $\left(x_{1} x_{2} x_{3}\right) x_{4}$. Let $h^{\prime}$ in $\mathscr{L}$ be written by using the operator $f^{\prime}$ replacing $f(l)$. Let

$$
\begin{aligned}
& \theta=x^{L} y^{U} z^{U}-x^{U} y^{U} z^{L}-x^{L} y^{L} z^{U}+x^{U} y^{L} z^{U} \\
& \bar{\theta}=x^{U} y^{L} z^{L}-x^{U} y^{U} z^{U}-x^{L} y^{L} z^{L}+x^{L} y^{U} z^{L} .
\end{aligned}
$$


We have:

$$
\begin{aligned}
\bar{R}\left(h^{\prime}\right)= & \left\{\left(w_{g}, w_{f^{\prime}}, x\right) \in \mathbb{R}^{6} \mid x^{L} \leq x \leq x^{U},\right. \\
& w_{f^{\prime}} \geq y^{U} z^{U} x+x^{U} z^{U} y+x^{U} y^{U} z-2 x^{U} y^{U} z^{U}, \\
& w_{f^{\prime}} \geq y^{U} z^{L} x+x^{L} z^{U} y+x^{L} y^{U} z-x^{L} y^{U} z^{L}-x^{L} y^{U} z^{U}, \\
& w_{f^{\prime}} \geq y^{U} z^{L} x+x^{L} z^{L} y+x^{L} y^{L} z-x^{L} y^{U} z^{L}-x^{L} y^{L} z^{L}, \\
& w_{f^{\prime}} \geq y^{L} z^{U} x+x^{U} z^{L} y+x^{U} y^{L} z-x^{U} y^{L} z^{U}-x^{U} y^{L} z^{L}, \\
& w_{f^{\prime}} \geq y^{L} z^{L} x+x^{U} z^{L} y+x^{L} y^{L} z-x^{U} y^{L} z^{L}-x^{L} y^{L} z^{L}, \\
& w_{f^{\prime}} \geq y^{L} z^{U} x+x^{L} z^{U} y+\left(\theta /\left(z^{U}-z^{L}\right)\right) z+ \\
& \left(-\left(\theta z^{L}\right) /\left(z^{U}-z^{L}\right)-x^{L} y^{U} z^{U}-x^{U} y^{L} z^{U}+x^{U} y^{U} z^{L}\right), \\
& w_{f^{\prime}} \leq y^{U} z^{L} x+x^{U} z^{L} y+x^{U} y^{U} z-2 x^{U} y^{U} z^{L}, \\
& w_{f^{\prime}} \leq y^{L} z^{L} x+x^{U} z^{U} y-x^{U} y^{L} z-x^{U} y^{L} z^{U}-x^{U} y^{L} z^{L}, \\
& w_{f^{\prime}} \leq y^{U} z^{U} x+x^{L} z^{U} y+x^{L} y^{L} z-x^{L} y^{U} z^{U}-x^{L} y^{L} z^{U}, \\
& w_{f^{\prime}} \leq y^{U} z^{U} x+x^{L} z^{L} y+x^{L} y^{U} z-x^{L} y^{U} z^{U}-x^{L} y^{U} z^{L}, \\
& w_{f^{\prime}} \leq y^{L} z^{U} x+x^{U} z^{U} y+x^{L} y^{L} z-x^{U} y^{L} z^{U}-x^{L} y^{L} z^{U}, \\
& w_{f^{\prime}} \leq y^{L} z^{L} x+x^{L} z^{L} y+\left(\bar{\theta} /\left(z^{L}-z^{U}\right)\right) z+ \\
& \left(-\left(\bar{\theta} z^{U}\right) /\left(z^{L}-z^{U}\right)-x^{U} y^{L} z^{L}-x^{L} y^{U} z^{L}+x^{U} y^{U} z^{U}\right), \\
& w_{g} \geq w_{f^{\prime}}^{L} x_{4}+x_{4}^{L} w_{f^{\prime}}-w_{f^{\prime}}^{L} x_{4}^{L}, w_{g} \geq w_{f^{\prime}}^{U} x_{4}+x_{4}^{U} w_{f^{\prime}}-w_{f^{\prime}}^{U} x_{4}^{U}, \\
& \left.w_{g} \leq w_{f^{\prime}}^{L} x_{4}+x_{4}^{U} w_{f^{\prime}}-w_{f^{\prime}}^{L} x_{4}^{U}, w_{g} \leq w_{f^{\prime}}^{U} x_{4}+x_{4}^{L} w_{f^{\prime}}-w_{f^{\prime}}^{U} x_{4}^{L}\right\} .
\end{aligned}
$$

Let $R(h)=\{(w, x) \mid \exists \mathbf{w}(w, \mathbf{w}, x) \in \bar{R}(h)\}$ be the projection of $\bar{R}$ and similarly let $R\left(h^{\prime}\right)$ be the projection of $\bar{R}\left(h^{\prime}\right)$. The hypotesis $\mathscr{R}(h) \subseteq R(h)$ is satisfied, hence, from Theorem 3.3, we have that $R\left(h^{\prime}\right) \subseteq R(h)$, i.e. $S_{3}$ is at least as tight as $S_{1}$.

\section{Computational assessment for the quadrilinear term}

Theorem 3.3 allows the comparison of some pairs of relaxations of quadrilinear terms, but it does not give an indication on the actual strength of the relaxations. We carried out numerical experiments to analyze the four convex relaxations in order to evaluate their relative tightness. Toward this aim, we generated a set of instances varying the signs of the bounds on the variables $x_{i}, i=1, \ldots, 4$. Missing cases on sign combinations are equivalent to covered cases by simple symmetry considerations. Instances were generated having the same initial width of the bound intervals for all variables, and then progressively, for $i=1,2,3$, reducing the width of the bound interval of $x_{i}$. This simulates a typical behavior of a sBB algorithm, which progressively reduces the size of the variable intervals. This reduction in the widths of the intervals is made preserving the signs of the bounds, changing a bound interval $\left[x_{i}^{L}, x_{i}^{U}\right]$ to $\left[x_{i}^{L}+1 / 2, x_{i}^{U}-1 / 2\right]$. Initial intervals $\left[x_{i}^{L}, x_{i}^{U}\right]$ were generated by considering the cases $x_{i}^{L}>0, x_{i}^{U}>0$, $x_{i}^{L}<0, x_{i}^{U}<0$ and $x_{i}^{L}<0, x_{i}^{U}>0$. Specifically, we set $x_{i}^{L}=1, x_{i}^{U}=3$ in the first case, $x_{i}^{L}=-3, x_{i}^{U}=$ -1 in the second case and $x_{i}^{L}=-1, x_{i}^{U}=1$ in the third case.

The comparison among the considered relaxations is made in terms of the volume of the corresponding enclosing polytopes. This method of comparison, introduced in [19], is independent of any objective function. Because exploiting envelopes for bilinear and trilinear terms leads to an increased number of variables, so that the obtained polytopes belong to $\mathbb{R}^{7}\left(S_{1}, S_{2}\right)$ and $\mathbb{R}^{6}\left(S_{3}, S_{4}\right)$ respectively, we project the polytopes on $\left(x, f(x):=x_{1} x_{2} x_{3} x_{4}\right) \in \mathbb{R}^{5}$ in order to compare the results. The projection is computed using the software CDD [9], that calculates projections in exact rational arithmetic. Then, the volume of each of the obtained projected polytopes is computed using the LRS code [4]. Again, the results are computed in exact rational arithmetic.

In Table 2 we report the values of the volumes of the polytopes corresponding to $S_{1}, S_{2}, S_{3}, S_{4}$ 
projected onto $\mathbb{R}^{5}$, obtained for each problem instance. We remark that the volumes are reported in fixedprecision decimal format for easier reading, although they were computed in exact rational arithmetic. For each variable $x_{i}$, the width of the bound interval $x_{i}^{U}-x_{i}^{L}$ and the sign of $x_{i}^{L}$ and $x_{i}^{U}$ are also listed.

As expected, reducing the width of the bounds on variables, the polytopes have decreasing volumes, while keeping the same relative size with respect to the others. Note that for $85 \%$ of the test instances, the smallest values of the volume are obtained with the relaxation corresponding to $S_{4}$. These values in $5 \%$ of instances are the same obtained with $S_{3}$ and with $S_{1}$ and are the same obtained with $S_{2}$ again in $5 \%$ of instances (other than the previous ones). On the remaining instances, in $5 \%$ of cases we get the same value for the volume of the four enveloping polytopes, in the other cases the smallest values are obtained with $S_{3}$. These results show that $S_{3}$ and $S_{4}$ always yield the best relaxations. It is interesting to remark that in some cases the lowest value is also reached exploiting bilinear relaxations, but we never find that $S_{1}$ and $S_{2}$ provide the lowest volumes. This confirms the Theorem 3.3 (Section 3): the best relaxations are obtained employing convex envelopes for trilinear terms and not just bilinear ones. Although our results depend on the particular bounds that we tried, we get a significant indication of the strength of the considered relaxations and their dependence on signs and widths of bounds.

We also compute the extreme points of $w=x_{1} x_{2} x_{3} x_{4}$ by considering all the combinations of bounds on the variables and we get the convex hull for these points. This gives the tightest linear approximation of the quadrilinear term, but it is not used in sBB codes because it cannot be expressed explicitly in function of the bounds on the variables. We use it as an approximation against which to compare the others. In Table 3 we report, for each of the considered relaxations, the ratios between the volume of the enveloping polytope projected onto $\mathbb{R}^{5}$ and the volume of the above approximation. According to the results in Table 2, we find the lowest ratios for the relaxation corresponding to $S_{4}$. Furthermore, for the $5 \%$ of the instances all the volumes are equal; for $5 \%$ of the instances other than the previous ones, $S_{1}, S_{3}$ and $S_{4}$ give the same volume of that used as reference. For the remaining cases, we find some instances (10\%) for which the polytope corresponding to $S_{4}$ is not only the tightest with respect to $S_{1}$, $S_{2}, S_{3}$, but is also equivalent to the polytope around the extreme points.

In order to easily analyze the overall pattern that is emerging in terms of variations of volumes of the enveloping polytopes, we report a graphical representation of the computational results. For each of the sign combinations, we plot 4 line graphs, one for each of the linearizations. Each line has 4 points, corresponding to the instances obtained by progressively tightening the bounds for the considered sign combination. These graphs suggest that the relaxation strength of quadrilinear terms follow different behaviors according to different bound widths/signs. We note that, after tightening the bounds on a variable, there are no examples where one curve goes from far below to far above another. This suggests that tightening the bounds has a comparable effect on the different relaxations.

The considered polytopes are expected to overlap. Given two polytopes, they share some common points or one is entirely contained in the other. We get a more precise information about tightness of the considered relaxations by checking relative containments of the corresponding (projected) polytopes. For each pair of polytopes $P, Q$, we check if $P$ is contained in $Q$ by checking that every extreme point of $P$ satisfies all the inequalities defining $Q$. Given a pair $P, Q$ such that the volume of $P$ is less than or equal to that of $Q$, we are able to estabilish if $Q$ contains $P$. This gives a stronger indication on the "dominance" of a relaxation with respect to another. The obtained results are reported in Table 4 . As expected from results in Table 2, relaxation $S_{4}$ gives a polytope which is the most frequently contained in the others. This polytope is always contained in that corresponding to $S_{1}$ and $S_{2}$. It is sometimes equivalent to these polytopes, specifically in the $20 \%$ and in the $15 \%$ of the cases respectively. $S_{3}$ also gives a polytope always contained or equivalent to that given by $S_{1}$. This is interesting, being $S_{1}$ currently the most used relaxation in implementations. For some instances (5\%, e.g. i_5), we find that all the polytopes are equivalent. 


\begin{tabular}{|c|c|c|c|c|c|c|c|c|c|c|c|c|}
\hline$\#$ & \multicolumn{2}{|c|}{$x_{1}$} & \multicolumn{2}{|c|}{$x_{2}$} & & 3 & & $c_{4}$ & $S_{1}$ & $S_{2}$ & $S_{3}$ & $S_{4}$ \\
\hline & $|\cdot|$ & $\operatorname{sign}$ & 1.1 & $\operatorname{sign}$ & $|\cdot|$ & $\operatorname{sign}$ & 1.1 & $\operatorname{sign}$ & & & & \\
\hline i_.1 & $\frac{1}{2}$ &,++ & 2 &,++ & 2 &,-+ & 2 &,-+ & 184.0444 & 98.2667 & 175.4074 & 98.2667 \\
\hline i_2 & 2 &,++ & 2 &,++ & 2 &,-+ & 2 &,-- & 242.7111 & 245.9202 & 223.4074 & 185.6000 \\
\hline i_3 & 2 &,++ & 2 &,-+ & 2 &,-+ & 2 &,-+ & 77.0370 & 77.0370 & 69.6889 & 77.0370 \\
\hline i_4 & 2 &,++ & 2 &,-+ & 2 &,-+ & 2 &,-- & 126.8148 & 203.7333 & 101.6889 & 126.8148 \\
\hline i_5 & 2 &,-+ & 2 &,-+ & 2 &,-+ & 2 &,-+ & 27.7333 & 27.7333 & 27.7333 & 27.7333 \\
\hline $\begin{array}{l}1.0 \\
\text { i. } 6\end{array}$ & 2 & $\begin{array}{l}-,+ \\
-,+\end{array}$ & 2 & $\begin{array}{l}-,+ \\
-,+\end{array}$ & 2 & $\begin{array}{l}-,+ \\
-,+\end{array}$ & 2 & $\begin{array}{l}-,+ \\
-,-\end{array}$ & 49.0667 & 77.0370 & 49.0667 & 49.0667 \\
\hline $\begin{array}{l}\text { i. } 7 \\
\text { in }\end{array}$ & 2 &,++ & 2 &,-+ & 2 &,--1 & 2 &,-+ & 184.8889 & 203.7333 & 175.4074 & 126.8148 \\
\hline $\begin{array}{l}1-1 \\
\text { i__8 }\end{array}$ & 2 & $\begin{array}{l}+,+ \\
+,+\end{array}$ & 2 &,-+ & 2 &,-- & 2 &,-+ & 245.3333 & 245.9202 & 223.4074 & 209.7778 \\
\hline i_9 & 2 &,-+ & 2 &,-- & 2 &,-- & 2 &,-+ & 184.8889 & 203.7333 & 175.4074 & 126.8148 \\
\hline i_ 10 & 2 &,-+ & 2 &,-- & 2 &,-- & 2 &,-- & 245.3333 & 245.9202 & 223.4074 & 209.7778 \\
\hline i_-11 & 2 &,-+ & 2 &,-+ & 2 &,-- & 2 &,-+ & 69.6889 & 77.0370 & 69.6889 & 49.0667 \\
\hline $\begin{array}{l}1.11 \\
\text { i.112 }\end{array}$ & 2 & $\begin{array}{l}-,+ \\
-,+\end{array}$ & 2 & $\begin{array}{l}-,+ \\
-,+\end{array}$ & $\begin{array}{l}2 \\
2\end{array}$ & -, - - & 2 & $\begin{array}{l}-,+ \\
-,-\end{array}$ & $\begin{array}{r}09.6889 \\
101.6889\end{array}$ & 98.2667 & $\begin{array}{r}09.6889 \\
101.6889\end{array}$ & 89.6000 \\
\hline $\begin{array}{l}\text { i_13 } \\
\text { i_ts }\end{array}$ & 2 &,++ & 2 &,++ & 2 &,++ & 2 &,-+ & 205.9795 & 245.9202 & 196.3487 & 185.6000 \\
\hline i_14 & 2 &,++ & 2 &,++ & 2 &,++ & 2 &,--1 & 275.3128 & 296.2436 & 249.6821 & 249.2667 \\
\hline i_15 & 2 &,++ & 2 &,++ & 2 &,-- & 2 &,-+ & 205.9795 & 245.9202 & 196.3487 & 185.6000 \\
\hline i_16 & 2 &,++ & 2 &,++ & 2 &,-- & 2 &,-- & 275.3128 & 296.2436 & 249.6821 & 249.2667 \\
\hline i_ 17 & 2 &,++ & 2 &,-- & 2 &.-- & 2 &,-+ & 205.9795 & 245.9202 & 196.3487 & 185.6000 \\
\hline i_18 & 2 &,++ & 2 &,-- & 2 &,-- & 2 &,-- & 275.3128 & 296.2436 & 249.6821 & 249.2667 \\
\hline $\begin{array}{l}\text { i_1 } 19 \\
\text { int }\end{array}$ & 2 &,-- & 2 &,-- & 2 &,-- & 2 &,-+ & 205.9795 & 245.9202 & 196.3487 & 185.6000 \\
\hline i__20 & 2 &,-- & 2 &,-- & 2 &,-- & 2 &,--1 & 275.3128 & 296.2436 & 249.6821 & 249.2667 \\
\hline i_ 21 & 1 &,++ & 2 &,++ & 2 &,-+ & 2 &,-+ & 72.5304 & 41.9111 & 69.2622 & 41.9111 \\
\hline i_ 22 & 1 &,++ & 2 &,++ & 2 &,-+ & 2 &,--1 & 93.8637 & 97.0978 & 86.5956 & 73.5556 \\
\hline i_ 23 & 1 &,++ & 2 &,-+ & 2 &,-+ & 2 &,-+ & 30.3147 & 30.3147 & 28.3733 & 30.3147 \\
\hline i_ 24 & 1 &,++ & 2 &,-+ & 2 &,-+ & 2 &,-+ & 48.4480 & 77.1504 & 41.7067 & 48.4480 \\
\hline i_ 25 & 1 &,-+ & 2 &,-+ & 2 &,-+ & 2 &,-+ & 6.9333 & 6.9333 & 6.9333 & 6.9333 \\
\hline i_ 26 & 1 &,-+ & 2 &,-+ & 2 &,-+ & 2 &,--1 & 12.2667 & 19.2593 & 12.2667 & 12.2667 \\
\hline i_ 27 & 1 &,++ & 2 &.-+ & 2 &.-- & 2 &,-+ & 72.0071 & 77.1504 & 69.2622 & 48.4480 \\
\hline i_ 28 & 1 &,++ & 2 &,-+ & 2 &,-- & 2 &,--1 & 92.8071 & 92.6308 & 86.5956 & 77.7387 \\
\hline $\begin{array}{l}\text { i. } 29 \\
\text { i. }\end{array}$ & 1 & T, T & 2 & -1 & 2 & $\therefore-$ & 2 & -+ & 46.2222 & 50.9333 & 43.8519 & 31.7037 \\
\hline $\begin{array}{l}1.29 \\
\text { i_ } 30\end{array}$ & 1 & $\begin{array}{l}-,+ \\
-,+\end{array}$ & 2 &,-- & 2 &,-- & 2 & $\begin{array}{l}-,+ \\
-,-\end{array}$ & $\begin{array}{l}40.2222 \\
61.3333\end{array}$ & 61.4800 & $\begin{array}{l}45.8519 \\
55.8519\end{array}$ & 52.4444 \\
\hline i_ 31 & 1 &,-+ & 2 &,-+ & 2 &,-- & 2 &,-+ & 17.4222 & 19.2593 & 17.4222 & 12.2667 \\
\hline i_32 & 1 &,-+ & 2 &,-+ & 2 &,-- & 2 &,-- & 25.4222 & 24.5667 & 25.4222 & 22.4000 \\
\hline $\begin{array}{l}1.32 \\
\text { i_ } 33\end{array}$ & 1 & $\begin{array}{l}-,+ \\
+,+\end{array}$ & 2 & $\begin{array}{l}-,+ \\
+,+\end{array}$ & 2 &,++ & 2 &,-+ & 84.2095 & 97.0978 & 80.5206 & 73.5556 \\
\hline $\begin{array}{l}\text { i. } 34 \\
\text { i. } 34\end{array}$ & 1 &,++ & 2 &,++ & 2 &,++ & 2 & $-\therefore-$ & 108.2095 & 114.5142 & 99.1873 & 93.0000 \\
\hline i_35 & 1 & $\begin{array}{l}T+1 \\
+,+\end{array}$ & 2 & $\begin{array}{l}T+1 \\
+,+\end{array}$ & 2 & $\begin{array}{l}T, T \\
-,-\end{array}$ & 2 &,-+ & 84.2095 & 97.0978 & 80.5206 & 73.5556 \\
\hline i_-36 & 1 &,++ & 2 &,++ & 2 &,-- & 2 &,--1 & 108.2095 & 114.5142 & 99.1873 & 93.0000 \\
\hline i_ 37 & 1 &,++ & 2 &,-- & 2 &,-- & 2 &,-+ & 84.2095 & 97.0978 & 80.5206 & 73.5556 \\
\hline i_38 & 1 &,++ & 2 &,-- & 2 &,-- & 2 &,-- & 108.2095 & 114.5142 & 99.1873 & 93.0000 \\
\hline $\begin{array}{l}1 \text { i_so } \\
\text { i__39 }\end{array}$ & 1 & $\begin{array}{l}+,+ \\
--,-\end{array}$ & 2 & -, - & 2 & -, - & 2 &,$- \overline{+}$ & 84.2095 & 97.0978 & 80.5206 & 73.5556 \\
\hline $\begin{array}{l}\text { i_ } 40 \\
\text { ind }\end{array}$ & 1 &,-- & 2 &,-- & 2 &,-- & 2 &,--1 & 108.2095 & 114.5142 & 99.1873 & 93.0000 \\
\hline i_41 & 1 &,++ & 1 &,++ & 2 &,-+ & 2 &,-+ & 27.9709 & 17.6104 & 26.6667 & 17.6104 \\
\hline i_ 42 & 1 &,++ & 1 &,++ & 2 &,-+ & 2 &,-- & 35.1376 & 36.4175 & 32.3333 & 28.2958 \\
\hline $\begin{array}{l}1-72 \\
\text { i } 43\end{array}$ & 1 &.+ & 1 & $\ldots+$ & 2 & $\ldots+$ & 2 & $\therefore+$ & 7.5787 & 7.5787 & 7.0933 & 7.5787 \\
\hline $\begin{array}{l}\begin{array}{l}1.43 \\
\text { i_.44 }\end{array}\end{array}$ & 1 & $\begin{array}{l}+,+ \\
+,+\end{array}$ & 1 & $\begin{array}{l}-,+ \\
-,+\end{array}$ & $\begin{array}{l}2 \\
2\end{array}$ & $\begin{array}{l}-,+ \\
-,+\end{array}$ & 2 & $\begin{array}{l}-,+ \\
-,-\end{array}$ & 12.1120 & 19.2876 & 10.4267 & 12.1120 \\
\hline i_ 45 & 1 &,-+ & 1 &,-+ & 2 &,-+ & 2 &,-+ & 1.7333 & 1.7333 & 1.7333 & 1.7333 \\
\hline 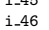 & 1 & $\begin{array}{l}-,+ \\
-,+\end{array}$ & 1 & $\begin{array}{l}-,+ \\
-,+\end{array}$ & 2 & $\begin{array}{l}-,+ \\
-,+\end{array}$ & 2 & $\begin{array}{l}-,+ \\
-,-\end{array}$ & $\begin{array}{l}1.0667 \\
\mathbf{3}\end{array}$ & $\begin{array}{l}1.333 \\
4.8148\end{array}$ & $\begin{array}{l}1.0667 \\
\mathbf{3}\end{array}$ & 3.0667 \\
\hline i_47 & 1 &,++ & 1 &,-+ & 2 &,--1 & 2 &,-+ & 18.0018 & 19.2876 & 17.3156 & 12.1120 \\
\hline $\begin{array}{l}\text { i_t } 48 \\
\text { i }\end{array}$ & 1 &,++ & 1 &,-+ & 2 &,-- & 2 &,-- & 23.2018 & 23.1577 & 21.6489 & 19.4347 \\
\hline i. 49 & 1 & $\ldots+$ & 1 & $\ldots-$ & 2 & $\ldots-$ & 2 & -+ & 18.0018 & 19.2876 & 17.3156 & 12.1120 \\
\hline i_50 & 1 &,-+ & 1 &,-- & 2 &,-- & 2 &,--1 & 23.2018 & 23.1577 & 21.6489 & 19.4347 \\
\hline $\begin{array}{l}\text { i__51 } \\
\text { i_to }\end{array}$ & 1 &,-+ & 1 &,-+ & 2 &,-- & 2 &,-+ & 4.3556 & 4.8148 & $\begin{array}{l}4.0409 \\
4.3556\end{array}$ & 3.0667 \\
\hline i_52 & 1 &,-+ & 1 &,-+ & 2 &,-- & 2 &,--1 & 6.3556 & 6.1417 & 6.3556 & 5.6000 \\
\hline i_53 & 1 &,++ & 1 &,++ & 2 &,++ & 2 &,-+ & 33.2785 & 36.4175 & 31.8323 & 28.2958 \\
\hline i_54 & 1 &,++ & 1 &,++ & 2 &,++ & 2 & $-1-$ & 41.1118 & 42.1904 & 37.8323 & 32.9208 \\
\hline i_55 & 1 &,++ & 1 &,++ & 2 &,--1 & 2 &,-+ & 33.2785 & 36.4175 & 31.8323 & 28.2958 \\
\hline i_56 & 1 & $\begin{array}{l}1 \\
+,+\end{array}$ & 1 & $\begin{array}{l}T+1 \\
+,+\end{array}$ & 2 &,-- & 2 &,--1 & 41.1118 & 42.1904 & 37.8323 & 32.9208 \\
\hline i_57 & 1 &,++ & 1 &,-- & 2 &,-- & 2 &,-+ & 33.2785 & 36.4175 & $\begin{array}{l}31.8323 \\
31.8323\end{array}$ & 28.2958 \\
\hline i_58 & 1 &,++ & 1 &,-- & 2 &,-- & 2 &,-- & 41.1118 & 42.1904 & 37.8323 & 32.9208 \\
\hline $\begin{array}{l}\text { i_59 } \\
\text { i. }\end{array}$ & 1 &,-- & 1 &,-- & 2 &,-- & 2 &,-+ & 33.2785 & 36.4175 & 31.8323 & 28.2958 \\
\hline $\begin{array}{l}\text { i. } 60 \\
\text { in }\end{array}$ & 1 &,-- & 1 &,-- & 2 &.- & 2 &,--1 & 41.1118 & 42.1904 & 37.8323 & 32.9208 \\
\hline i_61 & $\frac{1}{1}$ &,++ & $\frac{1}{1}$ & $\frac{1}{+,+}$ & $\frac{2}{1}$ & $\frac{1}{-,+}+$ & $\frac{2}{2}$ &,-+ & 6.9927 & 4.4026 & 6.6667 & 4.4026 \\
\hline i_62 & 1 &,++ & 1 &,++ & 1 &,-+ & 2 &,--1 & 8.7844 & 9.1044 & 8.0833 & 7.0740 \\
\hline i_63 & 1 &,++ & 1 &,-+ & 1 &,-+ & 2 &,-+ & 1.8947 & 1.8947 & 1.7733 & 1.8947 \\
\hline i. 64 & 1 &,++ & 1 &,-+ & 1 & $\ldots+$ & 2 &,-- & 3.0280 & 4.8219 & 2.6067 & 3.0280 \\
\hline $\begin{array}{l}1.64 \\
\text { i. } 65\end{array}$ & 1 & $\begin{array}{l}+,+ \\
-,+\end{array}$ & 1 & $\begin{array}{l}-,+ \\
-,+\end{array}$ & 1 & $\begin{array}{l}-,+ \\
-,+\end{array}$ & 2 & $\begin{array}{l}-,- \\
-,+\end{array}$ & $\begin{array}{l}3.0280 \\
0.4333\end{array}$ & $\begin{array}{l}4.8219 \\
0.4333\end{array}$ & $\begin{array}{c}2.0068 \\
0.4333\end{array}$ & $\begin{array}{l}3.0280 \\
0.4333\end{array}$ \\
\hline i_66 & 1 &,-+ & 1 &,-+ & 1 &,-+ & 2 &,--1 & 0.7667 & 1.2037 & 0.7667 & 0.7667 \\
\hline $\begin{array}{l}\text { i_ } 67 \\
\text { int }\end{array}$ & 1 &,++ & 1 &,-+ & 1 &,--1 & 2 &,-+ & 6.8539 & $\begin{array}{l}1.2001 \\
7.2208\end{array}$ & 6.6667 & 5.2947 \\
\hline i_68 & 1 &,++ & 1 &,-+ & 1 &,-- & 2 &,-- & 8.4872 & 8.8981 & 8.0833 & 7.4587 \\
\hline i_69 & 1 &,-+ & 1 & & 1 &,-- & 2 &,-+ & 6.8539 & 7.2208 & 6.6667 & 5.2947 \\
\hline $\begin{array}{l}\text { i_ } 70 \\
\text { in }\end{array}$ & 1 &,-+ & 1 &,-- & 1 & - & 2 &,--1 & 8.4872 & 8.8981 & 8.0833 & 7.4587 \\
\hline i.71 & 1 &,-+ & 1 &,-+ & 1 &,-- & 2 &,-+ & 1.7733 & 1.8947 & 1.7733 & 1.4333 \\
\hline $\begin{array}{l}1-1 \\
i\end{array} 72$ & 1 &,-+ & 1 &,-+ & 1 &,-- & 2 &,--1 & 2.6067 & 2.6194 & 2.6067 & 2.4000 \\
\hline i_73 & 1 &,++ & 1 & & 1 &,++ & 2 &,-+ & 12.3542 & 12.9119 & 11.8636 & 10.6573 \\
\hline $\begin{array}{l}\text { i. } 74 \\
\text { i }\end{array}$ & 1 &,++ & 1 &,++ & 1 &,++ & 2 & & 14.6459 & 15.3285 & 13.5303 & 12.1469 \\
\hline $\begin{array}{l}\text { i. } 75 \\
\text { i }\end{array}$ & 1 &,++ & 1 &,++ & 1 &,--1 & 2 &,-+ & 12.3542 & 12.9119 & 11.8636 & 10.6573 \\
\hline i_76 & 1 &,++ & 1 &,++ & 1 &,-- & 2 &,-- & 14.6459 & 15.3285 & 13.5303 & 12.1469 \\
\hline $\begin{array}{l}\text { i_ } 77 \\
\text { int }\end{array}$ & 1 & $\begin{array}{l}T,+ \\
+,+\end{array}$ & 1 &,-- & 1 &,-- & 2 &,-+ & 12.3542 & 12.9119 & 11.8636 & 10.6573 \\
\hline $\begin{array}{l}1-1 \\
\text { i. } 78\end{array}$ & 1 & $\begin{array}{l}+,+ \\
+,+\end{array}$ & 1 &,-- & 1 &,-- & 2 &,-+ & $\begin{array}{l}14.6459 \\
14.6459\end{array}$ & 15.3285 & $\begin{array}{l}11.0030 \\
13.5303\end{array}$ & 12.1469 \\
\hline $\begin{array}{l}\text { i_t9 } \\
\text { i }\end{array}$ & 1 &,-- & 1 &,-- & 1 &,-- & 2 &,-+ & 12.3542 & 12.9119 & 11.8636 & 10.6573 \\
\hline i_ 80 & 1 &,-- & 1 &,-- & 1 &,-- & 2 &,-- & 14.6459 & 15.3285 & 13.5303 & 12.1469 \\
\hline
\end{tabular}

Table 2: Volumes of the enveloping polytopes projected onto $\mathbb{R}^{5}$. For each $x_{i}, "|\cdot|$ " indicates the value of $x_{i}^{U}-x_{i}^{L}$, and "sign" indicates the pair of signs of $x_{i}^{L}, x_{i}^{U}$ respectively. We remark that the case of all positive signs is equivalent to that of all negative signs. 


\begin{tabular}{|c|c|c|c|c|c|c|c|c|c|c|c|c|}
\hline \multirow[t]{2}{*}{ \# } & \multicolumn{2}{|c|}{$x_{1}$} & & 2 & & 3 & & 4 & $S_{1}$ & $S_{2}$ & $S_{3}$ & $S_{4}$ \\
\hline & $|\cdot|$ & $\operatorname{sign}$ & 1.1 & $\operatorname{sign}$ & 1.1 & $\operatorname{sign}$ & 1.1 & $\operatorname{sign}$ & & & & \\
\hline i_1 & 2 &,++ & 2 &,++ & 2 &,-+ & 2 &,-+ & 2.0541 & 1.0967 & 1.9577 & 1.0967 \\
\hline i_2 & 2 &,++ & 2 &,++ & 2 &,-+ & 2 &,-- & 1.5271 & 1.5473 & 1.4057 & 1.1678 \\
\hline i_3 & 2 &,++ & 2 &,-+ & 2 &,-+ & 2 &,-+ & 1.5700 & 1.5700 & 1.4203 & 1.5700 \\
\hline i_4 & 2 &,++ & 2 &,-+ & 2 &,-+ & 2 &,-- & 1.4153 & 2.2738 & 1.1349 & 1.4153 \\
\hline i_5 & 2 &,-+ & 2 &,-+ & 2 &,-+ & 2 &,-+ & 1.0000 & 1.0000 & 1.0000 & 1.0000 \\
\hline i_6 & 2 &,-+ & 2 &,-+ & 2 &,-+ & 2 &,-- & 1.0000 & 1.5700 & 1.0000 & 1.0000 \\
\hline i_7 & 2 &,++ & 2 &,-+ & 2 &,-- & 2 &,-+ & 2.0635 & 2.2738 & 1.9577 & 1.4153 \\
\hline i_8 & 2 &,++ & 2 &,-+ & 2 &,-- & 2 &,-- & 1.5436 & 1.5473 & 1.4057 & 1.3199 \\
\hline i_-9 & 2 &,-+ & 2 &,-- & 2 &,-- & 2 &,-+ & 2.0635 & 2.2738 & 1.9577 & 1.4153 \\
\hline i_10 & 2 &,-+ & 2 &,-- & 2 &,-- & 2 &,-- & 1.5436 & 1.5473 & 1.4057 & 1.3199 \\
\hline i_11 & 2 &,-+ & 2 &,-+ & 2 &,-- & 2 &,-+ & 1.4203 & 1.5700 & 1.4203 & 1.0000 \\
\hline i_.12 & 2 &,-+ & 2 &,-+ & 2 &,-- & 2 &,-- & 1.1349 & 1.0967 & 1.1349 & 1.0000 \\
\hline i_13 & 2 &,++ & 2 &,++ & 2 &,++ & 2 &,-+ & 1.2960 & 1.5473 & 1.2354 & 1.1678 \\
\hline i_-14 & 2 &,++ & 2 &,++ & 2 &,++ & 2 &,-- & 1.4260 & 1.5344 & 1.2932 & 1.2911 \\
\hline i_15 & 2 &,++ & 2 &,++ & 2 &,-- & 2 &,-+ & 1.2960 & 1.5473 & 1.2354 & 1.1678 \\
\hline i_16 & 2 &,++ & 2 &,++ & 2 &,-- & 2 &,-- & 1.4260 & 1.5344 & 1.2932 & 1.2911 \\
\hline i_17 & 2 &,++ & 2 &,-- & 2 &,-- & 2 &,-+ & 1.2960 & 1.5473 & 1.2354 & 1.1678 \\
\hline i_-18 & 2 &,++ & 2 &,--- & 2 &,-- & 2 &,--1 & 1.4260 & 1.5344 & $\begin{array}{l}1.2932 \\
\text { S }\end{array}$ & 1.2911 \\
\hline i_19 & 2 &,-- & 2 &,-- & 2 &,-- & 2 &,-+ & 1.2960 & 1.5473 & 1.2354 & 1.1678 \\
\hline i. 20 & 2 &,-- & 2 &,-- & 2 &,-- & 2 &,-- & 1.4260 & 1.5344 & 1.2932 & 1.2911 \\
\hline i_21 & 1 &,++ & 2 &,++ & 2 &,-+ & 2 &,-+ & 1.8888 & 1.0914 & 1.8037 & 1.0914 \\
\hline i_ 22 & 1 &,++ & 2 &,++ & 2 &,-+ & 2 &,-- & 1.4728 & 1.5235 & 1.3587 & 1.1541 \\
\hline i. 23 & 1 &,++ & 2 &,-+ & 2 &,-+ & 2 &,-+ & 1.3219 & 1.3219 & 1.2372 & 1.3219 \\
\hline i. 24 & 1 &,++ & 2 &,-+ & 2 &,-+ & 2 &,-- & 1.2617 & 2.0091 & 1.0861 & 1.2617 \\
\hline i_ 25 & 1 &,-+ & 2 &,-+ & 2 &,-+ & 2 &,-+ & 1.0000 & 1.0000 & 1.0000 & 1.0000 \\
\hline i_26 & 1 &,-+ & 2 &,-+ & 2 &,-+ & 2 &,-- & 1.0000 & 1.5700 & 1.0000 & 1.0000 \\
\hline i__27 & 1 &,++ & 2 &,-+ & 2 &,-- & 2 &,-+ & 1.8752 & 2.0091 & 1.8037 & 1.2617 \\
\hline i_ 28 & 1 &,++ & 2 &,-+ & 2 &,-- & 2 &,-- & 1.4562 & 1.4534 & 1.3587 & 1.2197 \\
\hline i. 29 & 1 &,-+ & 2 &,-- & 2 &,-- & 2 &,-+ & 2.0635 & 2.2738 & 1.9577 & 1.4153 \\
\hline i_ 30 & 1 &,-+ & 2 &,-- & 2 &,-- & 2 &,-- & 1.5436 & 1.5473 & 1.4057 & 1.3199 \\
\hline i_31 & 1 &,-+ & 2 &,-+ & 2 &,-- & 2 &,-+ & 1.4203 & 1.5700 & 1.4203 & 1.0000 \\
\hline i_32 & 1 &,-+ & 2 &,-+ & 2 &,-- & 2 &,-- & 1.1349 & 1.0967 & 1.1349 & 1.0000 \\
\hline i_33 & 1 &,++ & 2 &,++ & 2 &,++ & 2 &,-+ & 1.3213 & 1.5235 & 1.2634 & 1.1541 \\
\hline 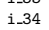 & 1 & $\begin{array}{l}T, T \\
+,+\end{array}$ & 2 & $\begin{array}{l}T \\
+,+\end{array}$ & 2 &,++ & 2 &,--1 & 1.4441 & 1.5282 & 1.3237 & 1.2411 \\
\hline$i_{-} 35$ & 1 &,++ & 2 &,++ & 2 &,-- & 2 &,-+ & 1.3213 & 1.5235 & 1.2634 & 1.1541 \\
\hline i_36 & 1 &,++ & 2 &,++ & 2 &,-- & 2 &,-- & 1.4441 & 1.5282 & 1.3237 & 1.2411 \\
\hline i_37 & 1 &,++ & 2 &,-- & 2 &,-- & 2 &,-+ & 1.3213 & 1.5235 & 1.2634 & 1.1541 \\
\hline i_38 & 1 &,++ & 2 &,-- & 2 &,-- & 2 &,-- & 1.4441 & 1.5282 & 1.3237 & 1.2411 \\
\hline i_39 & 1 &,-- & 2 &,-- & 2 &,-- & 2 &,-+ & 1.3213 & 1.5235 & 1.2634 & 1.1541 \\
\hline i__40 & 1 &,-- & 2 &,-- & 2 &,-- & 2 &,-- & 1.4441 & 1.5282 & 1.3237 & 1.2411 \\
\hline i_41 & 1 &,++ & 1 &,++ & 2 &,-+ & 2 &,-+ & 1.7195 & 1.0826 & 1.6393 & 1.0826 \\
\hline i_42 & 1 &,++ & 1 &,++ & 2 &,-+ & 2 &,-- & 1.4284 & 1.4804 & 1.3144 & 1.1502 \\
\hline i_43 & 1 &,++ & 1 &,-+ & 2 &,-+ & 2 &,-+ & 1.3219 & 1.3219 & 1.2372 & 1.3219 \\
\hline i. 44 & 1 &,++ & 1 &,-+ & 2 &,-+ & 2 &,--1 & 1.2617 & 2.0091 & 1.0861 & 1.2617 \\
\hline i_45 & 1 &,-+ & 1 &,-+ & 2 &,-+ & 2 &,-+ & 1.0000 & 1.0000 & 1.0000 & 1.0000 \\
\hline i_-46 & 1 &,-+ & 1 &,-+ & 2 &,-+ & 2 &,-- & 1.0000 & 1.5700 & 1.0000 & 1.0000 \\
\hline i_47 & 1 &,++ & 1 &,-+ & 2 &,-- & 2 &,-+ & 1.8752 & 2.0091 & 1.8037 & 1.2617 \\
\hline i_48 & 1 &,++ & 1 &,-+ & 2 &,-- & 2 &,-- & 1.4562 & 1.4534 & 1.3587 & 1.2197 \\
\hline i_49 & 1 &,-+ & 1 &,-- & 2 &,-- & 2 &,-+ & 1.8752 & 2.0091 & 1.8037 & 1.2617 \\
\hline i_50 & 1 &,-+ & 1 &,-- & 2 &,-- & 2 &,-- & 1.4562 & 1.4534 & 1.3587 & 1.2197 \\
\hline i_-51 & 1 &,-+ & 1 &,-+ & 2 &,-- & 2 &,-+ & 1.4203 & 1.5700 & 1.4203 & 1.0000 \\
\hline i_52 & 1 &,-+ & 1 &,-+ & 2 &,-- & 2 &,-- & 1.1349 & 1.0967 & 1.1349 & 1.0000 \\
\hline i_53 & 1 &,++ & 1 &,++ & 2 &,++ & 2 &,-+ & 1.3528 & 1.4804 & 1.2940 & 1.1502 \\
\hline i_54 & 1 &,++ & 1 &,++ & 2 &,++ & 2 &,-- & 1.4648 & 1.5032 & 1.3479 & 1.1730 \\
\hline i__55 & 1 &,++ & 1 &,++ & 2 &,--1 & 2 &,-+ & 1.3528 & 1.4804 & 1.2940 & 1.1502 \\
\hline i_56 & 1 &,++ & 1 &,++ & 2 &,-- & 2 &,-- & 1.4648 & 1.5032 & 1.3479 & 1.1730 \\
\hline i_57 & 1 &,++ & 1 &,-- & 2 &,-- & 2 &,-+ & 1.3528 & 1.4804 & 1.2940 & 1.1502 \\
\hline i_58 & 1 &,++ & 1 &,-- & 2 &,-- & 2 &,-- & 1.4648 & 1.5032 & 1.3479 & 1.1730 \\
\hline i_-59 & 1 &,-- & 1 &,-- & 2 &,-- & 2 &,-+ & 1.3528 & 1.4804 & 1.2940 & 1.1502 \\
\hline i_ 60 & 1 &,-- & 1 &,-- & 2 &,-- & 2 &,-- & 1.4648 & 1.5032 & 1.3479 & 1.1730 \\
\hline i_661 & 1 &,++ & 1 &,++ & 1 &,-+ & 2 &,-+ & 1.7195 & 1.0826 & 1.6393 & 1.0826 \\
\hline i_ 62 & 1 &,++ & 1 &,++ & 1 &,-+ & 2 &,-- & 1.4284 & 1.4804 & 1.3144 & 1.1502 \\
\hline i_63 & 1 &,++ & 1 &,-+ & 1 &,-+ & 2 &,-+ & 1.3219 & 1.3219 & 1.2372 & 1.3219 \\
\hline i_64 & 1 &,++ & 1 &,-+ & 1 &,-+ & 2 &,-- & 1.2617 & 2.0091 & 1.0861 & 1.2617 \\
\hline i_ 65 & 1 &,-+ & 1 &,-+ & 1 &,-+ & 2 &,-+ & 1.0000 & 1.0000 & 1.0000 & 1.0000 \\
\hline i_66 & 1 &,-+ & 1 &,-+ & 1 &,-+ & 2 &,-- & 1.0000 & 1.5700 & 1.0000 & 1.0000 \\
\hline i_-67 & 1 &,++ & 1 &,-+ & 1 &,-- & 2 &,-+ & 1.6854 & 1.7756 & 1.6393 & 1.3020 \\
\hline i_68 & 1 &,++ & 1 &,-+ & 1 &,-- & 2 &,-- & 1.3800 & 1.4469 & 1.3144 & 1.2128 \\
\hline i_69 & 1 &,-+ & 1 &,-- & 1 &,-- & 2 &,-+ & 1.6854 & 1.7756 & 1.6393 & 1.3020 \\
\hline i_-70 & 1 &,-+ & 1 &,-- & 1 &,-- & 2 &,-- & 1.3800 & 1.4469 & 1.3144 & 1.2128 \\
\hline 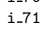 & 1 &,-+ & 1 &,-+ & 1 &,-- & 2 &,-++ & 1.2372 & 1.3219 & 1.2372 & 1.0000 \\
\hline i. 72 & 1 &,-+ & 1 &,-+ & 1 &,-- & 2 &,-- & 1.0861 & 1.0914 & 1.0861 & 1.0000 \\
\hline i. 73 & 1 &,++ & 1 &,++ & 1 &,++ & 2 &,-+ & 1.3855 & 1.4481 & 1.3305 & 1.1952 \\
\hline i_.74 & 1 &,++ & 1 &,++ & 1 &,++ & 2 &,-- & 1.4970 & 1.5668 & 1.3830 & 1.2416 \\
\hline i_.75 & 1 &,++ & 1 &,++ & 1 &,-- & 2 &,-+ & 1.3855 & 1.4481 & 1.3305 & 1.1952 \\
\hline i_-76 & 1 &,++ & 1 &,++ & 1 &,-- & 2 &,-- & 1.4970 & 1.5668 & 1.3830 & 1.2416 \\
\hline i_.77 & 1 &,++ & 1 &,-- & 1 &,-- & 2 &,-+ & 1.3855 & 1.4481 & 1.3305 & 1.1952 \\
\hline i. 78 & 1 &,++ & 1 &,-- & 1 &,-- & 2 &,-- & 1.4970 & 1.5668 & 1.3830 & 1.2416 \\
\hline i_.79 & 1 &,-- & 1 &,-- & 1 &,-- & 2 &,-+ & 1.3855 & 1.4481 & 1.3305 & 1.1952 \\
\hline i_ 80 & 1 &,-- & 1 &,-- & 1 &,-- & 2 &,-- & 1.4970 & 1.5668 & 1.3830 & 1.2416 \\
\hline
\end{tabular}

Table 3: Ratios of the volumes of the enveloping polytopes projected onto $\mathbb{R}^{5}$ and the volume of the convex hull of the extreme points of $w=x_{1} x_{2} x_{3} x_{4}$. For each $x_{i},|\cdot|=x_{i}^{U}-x_{i}^{L}$ and sign is the sign of $x_{i}^{L}$, $x_{i}^{U}$ respectively. We remark that the case of all positive signs is equivalent to that of all negative signs. 

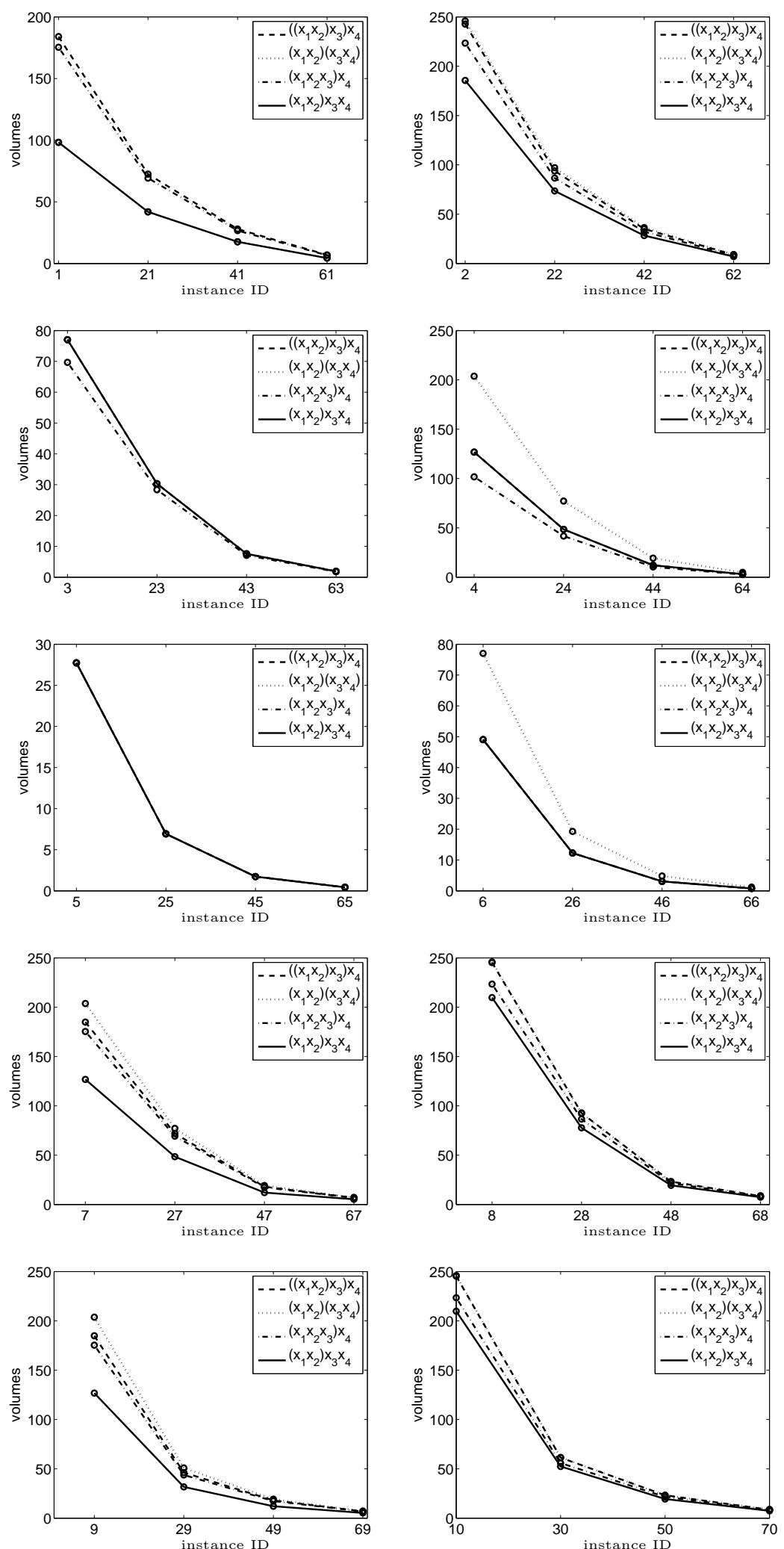

Figure 1: Volumes of enveloping polytopes corresponding to $S_{1}, S_{2}, S_{3}, S_{4}$. 

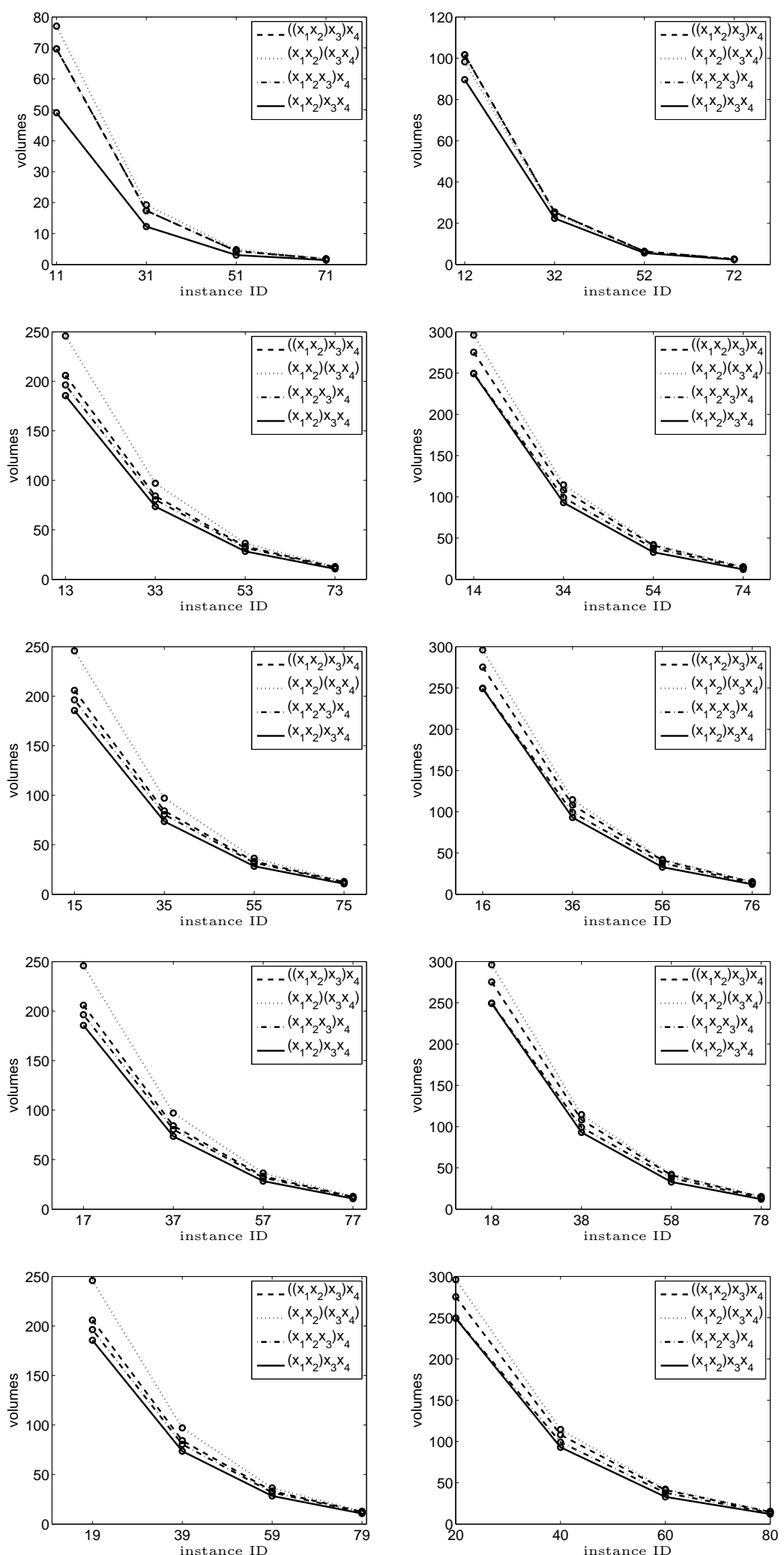

Figure 2: Volumes of enveloping polytopes corresponding to $S_{1}, S_{2}, S_{3}, S_{4}$. 


\begin{tabular}{|c|c|c|c|c|c|c|c|c|c|}
\hline \# & & $c_{1}$ & & $x_{2}$ & & $x_{3}$ & & $c_{4}$ & containments \\
\hline & $1 \cdot 1$ & $\operatorname{sign}$ & $|\cdot|$ & $\operatorname{sign}$ & $1 \cdot 1$ & $\operatorname{sign}$ & 1.1 & sign & Containments \\
\hline $\begin{array}{l}i-1 \\
i=2\end{array}$ & 2 &,++ & 2 & $\begin{array}{l}+,+ \\
+,+\end{array}$ & $\begin{array}{r}2 \\
2\end{array}$ &,-+ & 2 &,-+ & $S_{2} \equiv S_{4} \subseteq S_{1}, S_{3} \subseteq S_{1}$ \\
\hline $\begin{array}{l}1.2 \\
\text { i. } 3\end{array}$ & 2 & $\begin{array}{l}+,+ \\
+,+\end{array}$ & 2 & $\begin{array}{l}+,+ \\
-,+\end{array}$ & 2 & $\begin{array}{l}-,+ \\
-,+\end{array}$ & 2 &,-+ & $S_{3} \subseteq S_{1} \subset S_{4} \supseteqq S_{1} \equiv S_{2}, S_{4} \subseteq S_{4} \subseteq S_{2}$ \\
\hline i. 4 & 2 &,++ & 2 &,-+ & 2 &,-+ & 2 &,-- & $S_{3} \subseteq S_{1}^{1} \equiv S_{4}^{2} \subseteq S_{2}^{*}$ \\
\hline $\begin{array}{l}i .5 \\
i .6\end{array}$ & $\begin{array}{l}2 \\
2\end{array}$ & 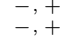 & $\begin{array}{l}2 \\
2\end{array}$ & $\begin{array}{l}-,+ \\
-,+\end{array}$ & ${ }_{2}^{2}$ & $\begin{array}{l}-,+ \\
-,+\end{array}$ & $\begin{array}{l}2 \\
2\end{array}$ & $\begin{array}{l}-,+ \\
-,-\end{array}$ & $\begin{array}{l}S_{1} \equiv{ }_{1} \equiv S_{2} \equiv S_{3} \equiv S_{3} \equiv S_{4} \equiv S_{4} \\
S_{3}\end{array}$ \\
\hline i. -7 & 2 &,++ & 2 &,-+ & 2 &,--1 & 2 &,-+ & $S_{3} \subseteq S_{1}, S_{4} \subseteq S_{1}, S_{4} \subseteq S_{2}$ \\
\hline $\begin{array}{lll}1.8 \\
i .\end{array}$ & 2 &,++ & 2 &,-+ & 2 &,-- & $\begin{array}{l}2 \\
2\end{array}$ &,-- & 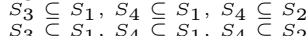 \\
\hline i. 10 & 2 &,-+ & 2 &,-- & 2 &,-- & 2 &,--1 & $S_{3} \subseteq S_{1}, S_{4} \subseteq S_{1}, S_{4} \subseteq S_{2}$ \\
\hline i. 11 & 2 &,-+ & 2 &,-+ & 2 &,-- & 2 &,-+ & $S_{3}, S_{4} \subseteq S_{2}$ \\
\hline 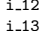 & $\begin{array}{l}2 \\
2\end{array}$ &,-+ & 2 &,-+ & ${ }_{2}^{2}$ &,--+ & $\begin{array}{l}2 \\
2\end{array}$ &,-- & $S_{3} S_{4}$ \\
\hline i. 14 & 2 &,++ & 2 &,++ & 2 & $\begin{array}{l}1, T \\
+,+\end{array}$ & 2 &,--1 & $S_{3} \subseteq S_{1}, S_{4} \subseteq$ \\
\hline i_15 & 2 &,++ & 2 &,++ & 2 &,-- & 2 &,-+ & $S_{3} \subseteq S_{1}, S_{4} \subseteq$ \\
\hline i. 16 & 2 &,++ & 2 &,++ & 2 &,-- & $\begin{array}{l}2 \\
2\end{array}$ & $\begin{array}{ll}-,- \\
-,+\end{array}$ & $S_{3} \subsetneq S_{S_{3}} \subset S_{1}, S_{4} \subsetneq S_{4} S_{1}, S_{S_{1}}$ \\
\hline i-1.18 & 2 &,++ & 2 &,,-- & 2 &,-- & 2 &,--1 & $S_{3} \subset S_{1}, S_{4} \subset S_{1}, S_{4} \subset$ \\
\hline i. 19 & 2 &,-- & 2 &,-- & 2 &,-- & 2 &,-+ & $S_{3} \subsetneq S_{1}, S_{4} \subseteq S_{1}, S_{4} \subsetneq S_{2}$ \\
\hline$\frac{1-21}{i-21}$ & $\frac{2}{1}$ &,++ & $\frac{2}{2}$ & $\frac{-1+}{+,+}$ & $\frac{2}{2}$ &,,-+ & $\frac{2}{2}$ &,-+ & $\frac{S_{3}}{S_{2} \equiv S_{4} \subseteq S_{1}, S 3 \subseteq S 1}$ \\
\hline i. 22 & 1 &,++ & 2 &,++ & 2 &,-+ & 2 &,-- & $S_{1}, S_{4}^{-} \subseteq S_{2}$ \\
\hline $\begin{array}{ll}1.23 \\
\text { i.24 }\end{array}$ & 1 & $\begin{array}{ll}+,+ \\
+,+\end{array}$ & 2 & $\begin{array}{l}-,++ \\
-,+\end{array}$ & 2 & $\begin{array}{ll}-,+ \\
-,+\end{array}$ & 2 &,-+ & $S_{4} \subset S_{2}$ \\
\hline i 225 & 1 &,-+ & 2 &,-+ & 2 &,-+ & 2 &,-+ & $S_{3} \equiv S_{4}$ \\
\hline $\begin{array}{l}i .26 \\
\text { i. } 27\end{array}$ & ${ }_{1}^{1}$ & 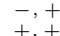 & ${ }_{2}^{2}$ & $\begin{array}{ll}-,+ \\
-,+\end{array}$ & ${ }_{2}^{2}$ &,-+ & ${ }_{2}^{2}$ &,-- & $S_{1} \equiv S_{3} \equiv$ \\
\hline $\begin{array}{l}1-27 \\
\text { i. } 28\end{array}$ & 1 &,++ & 2 &,-+ & 2 &,-- & 2 &,-- & $S_{3} \subseteq S_{1}, S_{4}$ \\
\hline i. 29 & 1 &,-+ & 2 &,-- & 2 &,-- & 2 &,-+ & $S_{3} \subseteq S_{1}, S_{4} \subsetneq$ \\
\hline $\begin{array}{l}i, 30 \\
i, 31\end{array}$ & $\begin{array}{l}1 \\
1\end{array}$ & $\begin{array}{l}-,+ \\
-,+\end{array}$ & ${ }_{2}^{2}$ &,-- & ${ }_{2}^{2}$ & 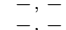 & $\begin{array}{l}2 \\
2\end{array}$ & $\begin{array}{ll}-,- \\
-,+\end{array}$ & $S_{3} \subseteq$ \\
\hline i 32 & 1 &,-+ & 2 &,-+ & 2 &,-- & 2 &,-- & $S_{4}^{4} \subseteq$ \\
\hline i_ 33 & &,++ & 2 & & 2 &,++ & 2 &,-+ & \\
\hline $\begin{array}{l}1.34 \\
i .35 \\
i\end{array}$ & $\begin{array}{l}1 \\
1\end{array}$ & $\begin{array}{l}+,+ \\
+,+\end{array}$ & ${ }_{2}^{2}$ & $\begin{array}{l}+,+ \\
+,+\end{array}$ & ${ }_{2}^{2}$ & $\begin{array}{l}+,+ \\
-,-\end{array}$ & $\begin{array}{l}2 \\
2\end{array}$ & $\begin{array}{l}-,- \\
-,+\end{array}$ & \\
\hline i_36 & 1 &,++ & 2 &,++ & 2 &,-- & 2 &,-- & $S_{3} \subseteq S_{1}, S_{4} \subseteq$ \\
\hline $\begin{array}{l}i .37 \\
i .38\end{array}$ & 1 & $\begin{array}{l}+,+ \\
+,+\end{array}$ & ${ }_{2}^{2}$ & $=-$, & ${ }_{2}^{2}$ &,-- & ${ }_{2}^{2}$ &,-+ & \\
\hline $\begin{array}{l}1.38 \\
i 39 \\
i 39\end{array}-10$ & 1 & $\stackrel{+,+}{-}$ & $\frac{2}{2}$ & $\begin{array}{ll}-,- \\
-,-\end{array}$ & $\frac{2}{2}$ &,-- & 2 &,-- & $S_{3} \subset S_{1}, S_{4}$ \\
\hline i. 40 & 1 &,-- & 2 &,-- & 2 &,-- & 2 &,--1 & $S_{3} \subseteq S_{1}, S_{4}^{4}$ \\
\hline i. 41 & 1 &,++ & 1 &,++ & 2 &,-+ & 2 &,-+ & $2 \equiv S_{4}$ \\
\hline $\begin{array}{l}i .42 \\
i .43\end{array}$ & 1 &,++ & 1 &,++ & 2 &,-+ & 2 &,-- & $C_{S} S 1$, \\
\hline i. 44 & 1 &,++ & 1 &,-+ & 2 &,-+1 & 2 &,--1 & $S_{3} \subset S_{1} \equiv S_{4} \subset S_{2}$ \\
\hline i. 45 & 1 &,-+ & 1 &,-+ & 2 &,-+ & 2 &,-+ & $S_{1}$ \\
\hline $\begin{array}{l}i .46 \\
i, 47\end{array}$ & 1 & $\begin{aligned}-,+ & \\
+,+ & 9\end{aligned}$ & 1 & $\begin{array}{ll}-,+ \\
-\end{array}$ & 2 &,-+ & ${ }_{2}^{2}$ &,-- & $S_{3} \subset C_{S}$ \\
\hline i. 48 & 1 &,++ & 1 &,-+ & 2 &,-- & 2 &,-- & $5=$ \\
\hline i. 49 & &,-+ & 1 &,-- & 2 &,-- & 2 &,-+ & \\
\hline $\begin{array}{l}1 \\
1.50 \\
i 51\end{array}$ & 1 &,-+ & 1 &,-- & 2 & $=,-$ & ${ }_{2}^{2}$ &,-- & $S_{3}$ \\
\hline i. 52 & 1 & 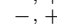 & 1 &,-+ & 2 &,-- & 2 &,-- & $\sigma^{4}$ \\
\hline i_ 53 & 1 &,++ & 1 &,++ & 2 &,++ & 2 &,-+ & $S_{3}$ \\
\hline i_ 54 & 1 &,++ & 1 &,++ & 2 &,++ & 2 &,-- & \\
\hline $\begin{array}{l}1.55 \\
\text { i. } 56 \\
-10\end{array}$ & 1 &,++ & & $\begin{array}{l}+,+ \\
+,+\end{array}$ & 2 &,-- & ${ }_{2}^{2}$ & 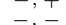 & $S_{3} \subset$ \\
\hline i_ 57 & 1 &,++ & 1 & 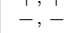 & 2 &,-- & 2 &,-+ & $S_{3}$ \\
\hline i_58 & &,++ & 1 &,-- & 2 & & $e^{2}+2$ & - & \\
\hline $\begin{array}{l}1.59 \\
\text { i.60 }\end{array}$ & 1 &,-- & 1 &,-- & ${ }_{2}^{2}$ &,-- & ${ }_{2}^{2}$ &,-+ & \\
\hline i_.61 & 1 & $\frac{1}{+,+t}$ & 1 & $\frac{1}{+,+}$ & 1 &,-+ & $\frac{2}{2}$ & $\frac{1}{-,++}$ & $\subset S_{1}, S 3 \subset S 1$ \\
\hline i. 62 & 1 &,+++ & 1 &,++ & 1 &,-+ & 2 & & \\
\hline i. & 1 &,++ & 1 &,-+ & 1 &,-+ & $\begin{array}{l}2 \\
2 \\
2\end{array}$ &,-+ & \\
\hline $\begin{array}{l}\text { i_ } 65 \\
\text { int }\end{array}$ & 1 & $\begin{array}{l}+,+ \\
-,+\end{array}$ & 1 &,-+ & 1 &,-+ & 2 & 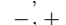 & $S_{3} \equiv S_{4}$ \\
\hline i. 66 & 1 &,-++ & 1 &,-+ & 1 &,-+ & 2 &,-- & $S_{1}^{1} \equiv S_{3}^{2}$ \\
\hline i. $\begin{array}{l}i .67 \\
i\end{array}$ & ${ }_{1}^{1}$ & $\begin{array}{l}+,+ \\
+,+\end{array}$ & 1 & $\begin{array}{l}-,+ \\
-,+\end{array}$ & $\begin{array}{l}1 \\
1\end{array}$ &,-- & 2 &,-+ & \\
\hline $\begin{array}{l}\begin{array}{l}1.68 \\
i .69\end{array} \\
i\end{array}$ & 1 & $\begin{array}{l}T, T \\
-,+\end{array}$ & 1 &,$- T$ & 1 &,-- & 2 &,-+ & $S_{3} \subset S_{1}$ \\
\hline i_ 70 & 1 &,-+ & 1 &,-- & 1 &,-- & 2 &,-- & $S_{3} \subseteq S$ \\
\hline i. -71 & &,-++ & 1 &,-+ & 1 &,-- & 2 &,-++ & \\
\hline $\begin{array}{l}1,1 / 2 \\
i=73\end{array}$ & 1 &,-+ & 1 &,++ & 1 & $\begin{array}{l}-,- \\
+,+\end{array}$ & $\begin{array}{l}2 \\
2\end{array}$ & $\begin{array}{l}-,- \\
-,+\end{array}$ & $S_{3} \subset \frac{S_{4}}{S} \frac{\mathrm{S}}{\mathrm{s}}$ \\
\hline i. 74 & 1 &,++ & 1 &,++ & 1 &,++ & 2 & & $S_{1}, S_{4}^{+} \bar{\subseteq}$ \\
\hline i. 75 & &,++ & &,++ & 1 & ; & 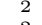 &,-++ & \\
\hline 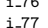 & 1 &,++ & 1 & $\stackrel{+,+}{-}$ & 1 &,-- & $\frac{2}{2}$ & $\begin{array}{l}-,- \\
-,+\end{array}$ & \\
\hline i. 78 & 1 & $\begin{array}{l}+,+ \\
+,+\end{array}$ & 1 &,-- & 1 &,-- & 2 &,--1 & $S_{3} \subseteq S_{1}$, \\
\hline $\begin{array}{l}i .79 \\
i .80\end{array}$ & $\begin{array}{l}1 \\
1\end{array}$ &,-- & $\begin{array}{l}1 \\
1\end{array}$ &,-- & $\begin{array}{l}1 \\
1\end{array}$ &,-- & $\begin{array}{l}2 \\
2\end{array}$ &,-+ & $S_{3} \subsetneq S_{1}, S_{4} \subseteq S_{1}, S_{4} \subseteq S_{2}$ \\
\hline
\end{tabular}

Table 4: Relative containments of the enveloping polytopes corresponding to $S_{1}, S_{2}, S_{3}, S_{4}$ projected onto $\mathbb{R}^{5}$. For each $x_{i},|\cdot|=x_{i}^{U}-x_{i}^{L}$ and sign is the sign of $x_{i}^{L}, x_{i}^{U} . S_{1}, S_{2}, S_{3}, S_{4}$ correspond to the term groupings $\left(\left(x_{1} x_{2}\right) x_{3}\right) x_{4},\left(x_{1} x_{2}\right)\left(x_{3} x_{4}\right),\left(x_{1} x_{2} x_{3}\right) x_{4},\left(x_{1} x_{2}\right) x_{3} x_{4}$, respectively. 


\section{Application to well-known problems}

We applied the obtained results to some well-known problems, namely the Molecular Distance Geometry Problem (MDGP) [17, 41] and the Hartree-Fock Problem (HFP) [18, 24]. Both problems, when cast in their mathematical programming formulation, are nonconvex polynomial NLPs with terms of degree up to four. Both can be solved to $\varepsilon$-optimality by means of the sBB algorithm.

\subsection{Molecular Distance Geometry Problem}

The MDGP is the problem of finding an embedding in $\mathbb{R}^{3}$ of a weighted graph $G$ such that all Euclidean distances between points in the embedding are the same as the corresponding edge weights in the graph. The main application is to find the three-dimensional structure of a molecule given a subset of the atomic distances (these are usually found using Nuclear Magnetic Resonance techniques) [6, 31].

Consider an undirected graph $G=(V, E)$ with weights $d: E \rightarrow \mathbb{R}_{+}$, where $V$ is the set of vertices (also called atoms), and $E$ is the set of weighted edges (also called inter-atomic distances). Let $N=|V|$ and $d_{i j}=d(\{i, j\})$ for $\{i, j\} \in E$. A solution of the MDGP is a set of points $x_{1}, \ldots, x_{N} \in \mathbb{R}^{3}$ satisfying

$$
\forall\{i, j\} \in E \quad\left\|x_{i}-x_{j}\right\|=d_{i j} .
$$

Each 3 -vector $x_{i}$ has components $\left(x_{i 1}, x_{i 2}, x_{i 3}\right)$, and we indicate the vector sequence $\left(x_{1}, \ldots, x_{N}\right)$ by $x$. The MDGP can be naturally cast as a continuous non-convex optimization problem $\min _{x} f(x)$ by minimizing the sum of the squared errors over each equation (2):

$$
f(x)=\sum_{\{i, j\} \in E}\left(\left\|x_{i}-x_{j}\right\|^{2}-d_{i j}^{2}\right)^{2} .
$$

Because each equation (2) must be satisfied, a candidate point $x$ is a solution of the MDGP if and only if $f(x)=0$. Note that (3) has a large number of local minima, so this is a difficult global-optimization problem.

When expanded, a typical term $\left(\left\|x_{i}-x_{j}\right\|^{2}-d_{i j}^{2}\right)^{2}$ of the MDGP objective function sum involves many quartic terms. We employ randomly generated MDGP instances as described in [15].

\subsection{Hartree-Fock Problem}

The quantum behaviour of atoms and molecules, in the absence of relativistic effects and any external time-dependent perturbations, is determined by the time-independent Schrödinger equation $H \Psi_{n}=E \Psi_{n}$, where $H$ (the Hamiltonian operator of the system) represents the total energy (kinetic + potential) of all the particles of the system. Analytical solutions for this equation are only possible for very simple systems. Hence, for the majority of problems of interest, one has to rely on some approximate model. In the Hartree-Fock (HF) model, the electrons in atoms and molecules move independently of each other, the motion of each one of the electrons being determined by the attractive electrostatic potential of the nuclei and by a repulsive average field due to all the other electrons of the system. In this model, the approximate solutions $\Phi_{n}$ of the Schrödinger equation are anti-symmetrized products of one-electron wave functions $\left\{\varphi_{i}\right\}$ (also called orbitals), which are solutions of the Hartree-Fock (HF) equations for the system under study. Since each orbital $\varphi_{i}$ can be expanded in a complete basis set $\left\{\chi_{s}\right\}_{s=1}^{\infty}$, we can transform the HF equations into a less cumbersome form by only considering a finite subset $\left\{\chi_{s} \mid s \leq b\right\}$ of the basis, and we use it to approximate the orbitals. We define the Hartree-Fock Problem (HFP) as the problem of finding a set of coefficients $c_{s i}$ such that the $\bar{\varphi}_{i}:=\sum_{s \leq b} c_{s i} \chi_{s}$ are the best possible approximations of the spatial orbitals. Thus, the decision variables of this mathematical programming problem are the coefficients $c_{s i}$. The objective function (quality of the approximation) is given by a 
suitable energy function $E$ associated with the approximating set $\left\{\bar{\varphi}_{i}\right\}$, which is constrained to be an orthonormal set:

$$
\begin{array}{ll}
\min & E(c) \\
\text { s.t. } & \left\langle\sum_{s \leq b} c_{s i} \chi_{s}, \sum_{s \leq b} c_{s j} \chi_{s}\right\rangle=\delta_{i j} \quad \forall i \leq j \leq n \\
& c^{L} \leq c \leq c^{U},
\end{array}
$$

where $\delta$ is the Kronecker delta function. We can readily see that the orthonormality constraints are quadratic in the decision variables $c$. Moreover, it turns out that for closed-shell atomic systems, the objective function is a quartic expression of the decision variables $c$.

\subsection{Bound evaluation algorithm}

The natural application of tight lower bounds computed through a convex relaxation is within the sBB algorithm. In order to quickly assess the quality of our proposed alternative bound for quadrilinear terms on the MDGP and HFP without having to implement a full sBB framework, we implemented (using AMPL [8]) a simplified "partial sBB" algorithm which, at each branching step, only records the most promising node and discards the other, thus exploring a single branch up to a leaf. This corresponds to well-known "diving heuristics" employed in integer linear programming. In Algoritm 1, $P$ is a mathematical program defined as $\min \left\{f(x) \mid g(x) \leq 0, x^{L} \leq x \leq x^{U}\right\}$ with decision variables $x \in \mathbb{R}^{n} \cap\left[x^{L}, x^{U}\right]$, objective function $f: \mathbb{R}^{n} \rightarrow \mathbb{R}$ and constraints $g: \mathbb{R}^{n} \rightarrow \mathbb{R}^{m}$.

Algorithm 1 can be considered as a heuristic solution algorithm for nonconvex NLPs, whose purpose is that of assessing the quality of a lower bound instead of that of the incumbent. We use a very simple branching strategy (the variable index $i$ maximizing $\left|x_{i}^{*}-\bar{x}_{i}\right|$ ) and terminate either on iteration limit or on reaching a node that is infeasible or that contains the global optimum.

$P$ is solved by SNOPT [10], and its (linear) convex relaxations $R_{0}, R_{1}$ by CPLEX [13]. $R_{0}, R_{1}$ are constructed automatically (by the ROSE software [22]) in the four different ways corresponding to $S_{1}-S_{4}$. The algorithm implemented in ROSE for constructing the convex relaxation is similar to the symbolic reformulation algorithm in [37]. First, each nonlinear term is replaced by an additional variable, and a defining constraint "additional variable $=$ nonlinear term" is added to the problem. In a second stage, each defining constraint is replaced by a convex relaxation. The different associativity precedences in $S_{1}-S_{4}$ yield different defining constraints and in turn different convex relaxations. For the sake of completeness, in the following we report the convex envelopes for piecewice/convex/concave monomials with the respective linear relaxations.

- The concave univariate function $f\left(x_{j}\right)$ is replaced by a variable $x_{i}$ and two inequalities are added to the problem relaxation: the function itself and the secant:

$$
\begin{aligned}
& x_{i} \leq f\left(x_{j}\right) \\
& x_{i} \geq f\left(x_{j}^{L}\right)+\frac{f\left(x_{j}^{U}\right)-f\left(x_{j}^{L}\right)}{x_{j}^{U}-x_{j}^{L}}\left(x_{j}-x_{j}^{L}\right) .
\end{aligned}
$$

Constraint (4) is a nonlinear over-estimator which is replaced in our implementation by a predetermined number of tangents to $f$ at various given points.

- The convex univariate function $f\left(x_{j}\right)$ is replaced by a variable $x_{i}$ and two inequalities are added to the problem relaxation:

$$
\begin{aligned}
& x_{i} \leq f\left(x_{j}^{L}\right)+\frac{f\left(x_{j}^{U}\right)-f\left(x_{j}^{L}\right)}{x_{j}^{U}-x_{j}^{L}}\left(x_{j}-x_{j}^{L}\right) \\
& x_{i} \geq f\left(x_{j}\right) .
\end{aligned}
$$

Constraint (7) is a nonlinear under-estimator which is replaced in our implementation by a predetermined number of tangents to $f$ at various given points. 


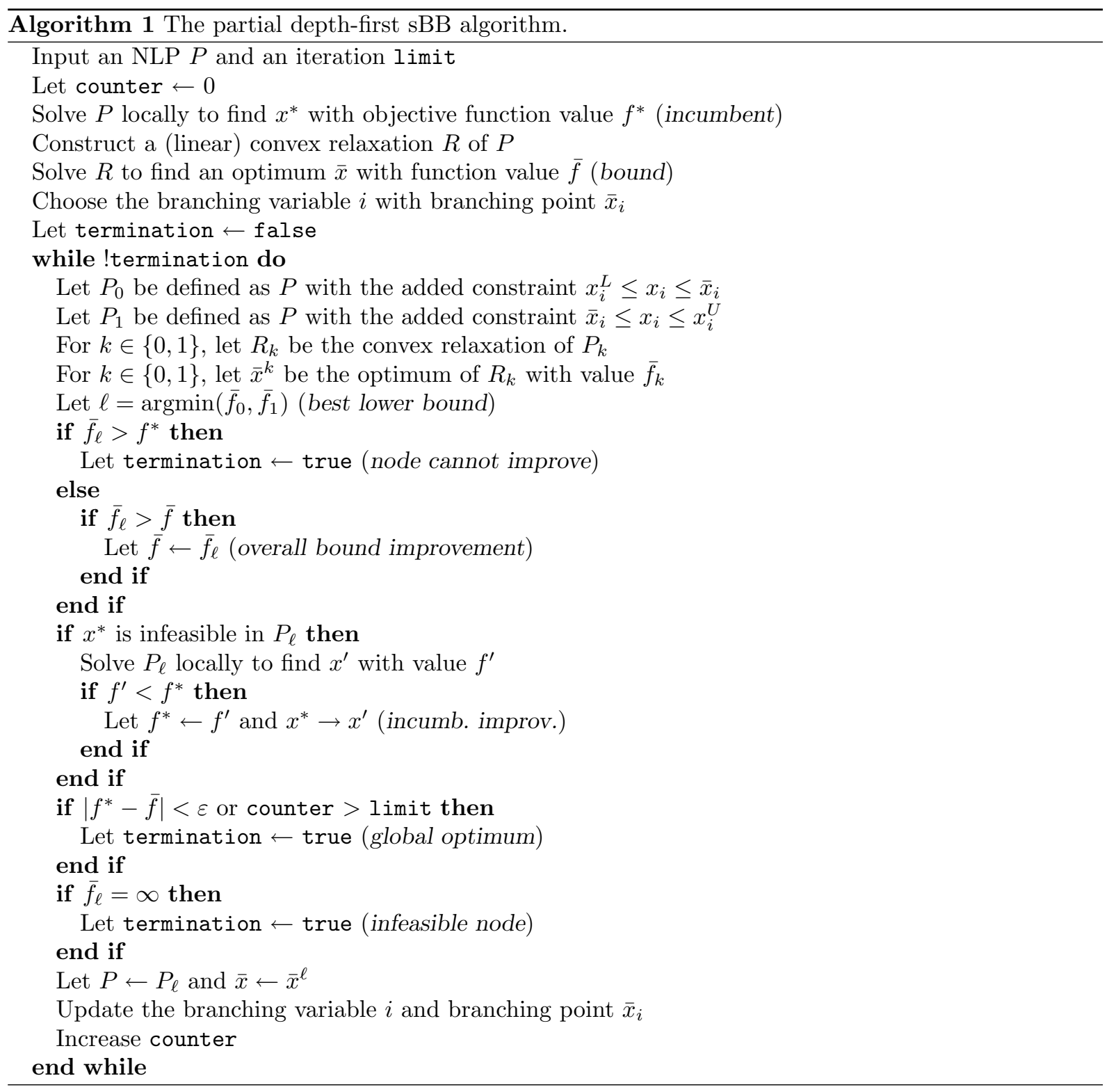

- The term $x_{j}^{2 k}$ for any $k \in \mathbb{N}$ is replaced by a variable $x_{i}$ and treated as a convex univariate function.

- The term $x_{j}^{2 k+1}$ for any $k \in \mathbb{N}$ is replaced by a variable $x_{i}$ and can be convex, concave, or piecewise convex and concave with a turning point at 0 . If the range of $x_{j}$ does not include 0 , the function is convex or concave and falls into a category described above. Otherwise, the convex/concave envelope is given in [26]; a tight linear relaxation is given by:

$$
\begin{aligned}
& x_{i} \geq\left(x_{j}^{L}\right)^{2 k+1}\left(1+T_{k}\left(\frac{x_{j}}{x_{j}^{L}}-1\right)\right) \\
& x_{i} \leq\left(x_{j}^{U}\right)^{2 k+1}\left(1+T_{k}\left(\frac{x_{j}}{x_{j}^{U}}-1\right)\right) \\
& x_{i} \geq(2 k+1)\left(x_{j}^{U}\right)^{2 k} x_{j}-2 k\left(x_{j}^{U}\right)^{2 k+1} \\
& x_{i} \leq(2 k+1)\left(x_{j}^{L}\right)^{2 k} x_{j}-2 k\left(x_{j}^{L}\right)^{2 k+1},
\end{aligned}
$$

where $T_{k}=\frac{t_{k}^{2 k+1}-1}{t_{k}-1}$ and the coefficients $t_{k}$ are given for the first few values of $k$ 's in the table below 


\begin{tabular}{||l||c|c|c|c||}
\hline \hline Instance & $S_{1}$ & $S_{2}$ & $S_{3}$ & $S_{4}$ \\
\hline lavor6 & -1006.75 & -1839.21 & -1006.75 & $\mathbf{- 9 9 0 . 1 6 7}$ \\
lavor7 & -1285.67 & -1279.88 & $\mathbf{- 1 1 7 5 . 9 5}$ & -1216.91 \\
lavor8 & -1711.27 & -1694.56 & -1718.41 & $\mathbf{- 1 6 7 1 . 0 9}$ \\
lavor10 & -3149.29 & -3172.05 & -3007.41 & $\mathbf{- 2 7 5 5 . 0 4}$ \\
\hline beryllium & -24.2038 & $\mathbf{- 2 2 . 4 6 3 9}$ & -24.2038 & $\mathbf{- 2 3 . 8 6 7 7}$ \\
neon & -683.034 & -619.238 & -651.045 & $\mathbf{- 5 0 8 . 0 6 1}$ \\
\hline \hline
\end{tabular}

Table 5: Results obtained by running Alg. 1 on MDGP and HFP instances.

(see [26] for details).

\begin{tabular}{c|c||l|c}
$k$ & $t_{k}$ & $k$ & $r_{k}$ \\
\hline 1 & -0.5000000000 & 6 & -0.7721416355 \\
2 & -0.6058295862 & 7 & -0.7921778546 \\
3 & -0.6703320476 & 8 & -0.8086048979 \\
4 & -0.7145377272 & 9 & -0.8223534102 \\
5 & -0.7470540749 & 10 & -0.8340533676
\end{tabular}

\subsection{Computational results}

Table 5 shows the results obtained by running Algorithm 1 on four MDGP and two HFP instances. We report the lower bounds obtained with the four relaxations as per $S_{1}-S_{4}$. On all the MDGP instances the best lower bound is that obtained with a relaxation involving a trilinear envelope. In particular, $S_{4}$ gives the tightest bound for most cases, and this bound is significantly better than the values obtained with bilinear relaxations on the largest MDGP instance. On the first HFP instance, we found a good bound using a relaxation based on bilinear envelopes. We never found that the first relaxation, which is currently the most used in sBB implementations, gives the best bounds. The CPU time taken to solve each of the different relaxations is about the same.

These results confirm the results of the previous sections and suggest that they can be used to configure a sBB algorithm to be efficiently applied to mathematical programs containing quadrilinear terms.

\section{Conclusion}

This paper focuses on convex relaxations of quadrilinear terms $x_{1} x_{2} x_{3} x_{4}$. We computationally and mathematically evaluated four linear relaxations, showing that the tightest one can be obtained by combining the convex envelope of a trilinear term and that of a bilinear term. Our results can be exploited in a sBB algorithm to compute tight bounds. Our mathematical result can be applied to compare relaxations of more general problems.

\section{acknowledgements}

We thank Komei Fukuda for helping us to use his code cdd to compute, in exact arithmetic, projections of polytopes and extreme point representations of polytopes given via inequalities. We thank Jesús De Loera for advising us to use David Avis' code lrs, and in turn we thank David Avis for lrs, which we used to compute exact volumes of polytopes given via extreme points. The first and the third author gratefully acknowledge financial support under ANR grant 07-JCJC-0151. 


\section{References}

[1] C.S. Adjiman. Global Optimization Techniques for Process Systems Engineering. PhD thesis, Princeton University, 1998.

[2] C.S. Adjiman, S. Dallwig, C.A. Floudas, and A. Neumaier. A global optimization method, $\alpha$ BB, for general twice-differentiable constrained NLPs: I. Theoretical advances. Computers $\&$ Chemical Engineering, 22(9):1137-1158, 1998.

[3] F.A. Al-Khayyal and J.E. Falk. Jointly constrained biconvex programming. Mathematics of Operations Research, 8(2):273-286, 1983.

[4] D. Avis. lrs. cgm.cs.mcgill.ca/ avis/C/lrs.html.

[5] P. Belotti, J. Lee, L. Liberti, F. Margot, and A. Wächter. Branching and bounds tightening techniques for non-convex MINLP, IBM Research Report RC24620, 2008.

[6] G.M. Crippen and T.F. Havel. Distance Geometry and Molecular Conformation. Wiley, New York, 1988.

[7] C. Floudas. Personal communication, 2007.

[8] R. Fourer and D. Gay. The AMPL Book. Duxbury Press, Pacific Grove, 2002.

[9] K. Fukuda. cdd. www.ifor.math.ethz.ch/ fukuda/cdd_home/cdd.html.

[10] P.E. Gill. User's guide for SNOPT version 7. Systems Optimization Laboratory, Stanford University, California, 2006.

[11] C.E. Gounaris and C.A. Floudas. Tight convex underestimators for $C^{2}$-continuous problems: I. Multivariate functions. Journal of Global Optimization, 42(1):69-89, 2008.

[12] C.E. Gounaris and C.A. Floudas. Tight convex underestimators for $C^{2}$-continuous problems: I. Univariate functions. Journal of Global Optimization, 42(1):51-67, 2008.

[13] ILOG. ILOG CPLEX 11.0 User's Manual. ILOG S.A., Gentilly, France, 2008.

[14] M. Jach, D. Michaels, and R. Weismantel. The convex envelope of (n-1)-convex functions. SIAM Journal on Optimization, 19(3):1451-1466, 2008.

[15] C. Lavor. On generating instances for the molecular distance geometry problem. In Liberti and Maculan [25], pages 405-414.

[16] C. Lavor, L. Liberti, and N. Maculan. Computational experience with the molecular distance geometry problem. In J. Pintér, editor, Global Optimization: Scientific and Engineering Case Studies, pages 213-225. Springer, Berlin, 2006.

[17] C. Lavor, L. Liberti, and N. Maculan. Molecular distance geometry problem. In C. Floudas and P. Pardalos, editors, Encyclopedia of Optimization, pages 2305-2311. Springer, New York, 2 edition, 2008.

[18] C. Lavor, L. Liberti, N. Maculan, and M.A. Chaer Nascimento. Solving Hartree-Fock systems with global optimization metohds. Europhysics Letters, 5(77):50006p1-50006p5, 2007.

[19] J. Lee and W.D. Morris, Jr. Geometric comparison of combinatorial polytopes. Discrete Applied Mathematics, 55(2):163-182, 1994.

[20] L. Liberti. Comparison of convex relaxations for monomials of odd degree. In I. Tseveendorj, P.M. Pardalos, and R. Enkhbat, editors, Optimization and Optimal Control. World Scientific, 2003.

[21] L. Liberti. Writing global optimization software. In Liberti and Maculan [25], pages 211-262. 
[22] L. Liberti, S. Cafieri, and F. Tarissan. Reformulations in mathematical programming: a computational approach. In A.-E. Hassanien, A. Abraham, F. Herrera, W. Pedrycz, A. Carvalho, P. Siarry, and A. Engelbrecht, editors, Foundations on Computational Intelligence, Studies in Computational Intelligence. Springer, Berlin, to appear.

[23] L. Liberti, C. Lavor, N. Maculan, and F. Marinelli. Double variable neighbourhood search with smoothing for the molecular distance geometry problem. Journal of Global Optimization, doi $10.1007 / \mathrm{s} 10898-007-9218-1$.

[24] L. Liberti, C. Lavor, M.A. Chaer Nascimento, and N. Maculan. Reformulation in mathematical programming: an application to quantum chemistry. Discrete Applied Mathematics, doi:10.1016/j.dam.2007.08.044.

[25] L. Liberti and N. Maculan, editors. Global Optimization: from Theory to Implementation. Springer, Berlin, 2006.

[26] L. Liberti and C.C. Pantelides. Convex envelopes of monomials of odd degree. Journal of Global Optimization, 25:157-168, 2003.

[27] L. Liberti, P. Tsiakis, B. Keeping, and C.C. Pantelides. ooOPPS. Centre for Process Systems Engineering, Chemical Engineering Department, Imperial College, London, UK, 2001.

[28] G.P. McCormick. Computability of global solutions to factorable nonconvex programs: Part I Convex underestimating problems. Mathematical Programming, 10:146-175, 1976.

[29] C.A. Meyer and C.A. Floudas. Trilinear monomials with positive or negative domains: Facets of the convex and concave envelopes. In C.A. Floudas and P.M. Pardalos, editors, Frontiers in Global Optimization, pages 327-352. Kluwer Academic Publishers, Amsterdam, 2003.

[30] C.A. Meyer and C.A. Floudas. Trilinear monomials with mixed sign domains: Facets of the convex and concave envelopes. Journal of Global Optimization, 29:125-155, 2004.

[31] A. Neumaier. Molecular modeling of proteins and mathematical prediction of protein structure. SIAM Review, 39:407-460, 1997.

[32] A. Rikun. A convex envelope formula for multilinear functions. Journal of Global Optimization, 10(4):425-437, 1997.

[33] H.S. Ryoo and N.V. Sahinidis. A branch-and-reduce approach to global optimization. Journal of Global Optimization, 8(2):107-138, March 1996.

[34] H.S. Ryoo and N.V. Sahinidis. Analysis of bounds for multilinear functions. Journal of Global Optimization, 19:403-424, 2001.

[35] N.V. Sahinidis and M. Tawarmalani. BARON 7.2.5: Global Optimization of Mixed-Integer Nonlinear Programs, User's Manual, 2005.

[36] E.M.B. Smith. On the Optimal Design of Continuous Processes. PhD thesis, Imperial College of Science, Technology and Medicine, University of London, October 1996.

[37] E.M.B. Smith and C.C. Pantelides. A symbolic reformulation/spatial branch-and-bound algorithm for the global optimisation of nonconvex MINLPs. Computers \& Chemical Engineering, 23:457-478, 1999.

[38] F. Tardella. Existence and sum decomposition of vertex polyhedral convex envelopes. Optimization Letters, 2:363-375, 2008.

[39] M. Tawarmalani and N. Sahinidis. Convex extensions and envelopes of semi-continuous functions. Mathematical Programming, 93(2):247-263, 2002. 
[40] M. Tawarmalani and N.V. Sahinidis. Global optimization of mixed integer nonlinear programs: A theoretical and computational study. Mathematical Programming, 99:563-591, 2004.

[41] J.-M. Yoon, Y. Gad, and Z. Wu. Mathematical modeling of protein structure using distance geometry. Technical Report TR00-24, Dept. Comput. Applied Maths, Rice University, Houston, 2000. 